This item was submitted to Loughborough's Research Repository by the author.

Items in Figshare are protected by copyright, with all rights reserved, unless otherwise indicated.

\title{
Augmented and virtual reality in construction: drivers and limitations for industry adoption
}

PLEASE CITE THE PUBLISHED VERSION

https://doi.org/10.1061/(ASCE)CO.1943-7862.0001844

PUBLISHER

ASCE

VERSION

AM (Accepted Manuscript)

\section{PUBLISHER STATEMENT}

This material may be downloaded for personal use only. Any other use requires prior permission of the American Society of Civil Engineers. This material may be found at https://ascelibrary.org/doi/abs/10.1061/(ASCE)CO.1943-7862.0001844.

\section{LICENCE}

\section{All Rights Reserved}

\section{REPOSITORY RECORD}

Delgado, Juan Manuel Davila, Lukumon Oyedele, Beach Thomas, and Peter Demian. 2020. "Augmented and Virtual Reality in Construction: Drivers and Limitations for Industry Adoption”. Loughborough University. https://hdl.handle.net/2134/11871366. 


\title{
Augmented and Virtual Reality in Construction: drivers and limitations for industry adoption
}

1. Juan Manuel Davila Delgado, Big Data Enterprise and Artificial Intelligence Laboratory, University of West of England Bristol, Coldharbour Lane, Bristol, BS16 1WD, UK, manuel.daviladelgado@uwe.ac.uk

2. Lukumon Oyedele ${ }^{1}$, Big Data Enterprise and Artificial Intelligence Laboratory, University of West of England Bristol, Coldharbour Lane, Bristol, BS16 1WD, UK, 1.oyedele@uwe.ac.uk

3. Thomas Beach, School of Engineering, Cardiff University, The Parade, Cardiff, CF24 3AA, UK, beachth@ cardiff.ac.uk

4. Peter Demian, School of Architecture, Building and Civil Engineering, Loughborough University, Loughborough LE11 3TU, UK, p.demian@lboro.ac.uk

1. Corresponding author.

\begin{abstract}
Augmented and virtual reality have the potential to provide a step-change in productivity in the construction sector; however, the level of adoption is very low. This paper presents a systematic study of the factors that limit and drive adoption in the construction sectorspecific context. A mixed research method was employed, combining qualitative and quantitative data collection and analysis. Eight focus groups with 54 experts and an online questionnaire were conducted. Forty-two limiting and driving factors were identified and ranked. Principal Component Analysis was conducted to group the identified factors into a smaller number of factors based on correlations. Four types of limiting factors and four types of driving factors were identified. The main limitation of adoption is that AR and VR technologies are regarded as expensive and immature technologies that are not suitable for engineering and construction. The main drivers are that AR and VR enable improvements in project delivery and provision of new and better services. This study provides valuable insights to stakeholders to devise actions that mitigate the limiting factors, and that boosts the driving factors. This is one of the first systematic studies that presents a detailed analysis of the factors that limit and drive adoption of AR and VR in the construction industry. The main contribution to knowledge of this study is that it grouped and characterized a myriad of limiting and driving factors into easily understandable categories; so that, the limiting factors can be effectively mitigated, and the driving factors potentiated. Also, a roadmap with specific short term and medium-term actions for improving adoption has been outlined.
\end{abstract}

Keywords: Augmented Reality, Virtual Reality, Construction, Architecture, Engineering, Limitations, Drivers, Adoption Roadmap. 


\section{Introduction}

Augmented Reality (AR) and Virtual Reality (VR) are visualization technologies that are dramatically changing the way humans interact with visual information. AR and VR technologies are becoming widespread, and every industry will be affected by the rapid adoption of these technologies. AR and VR technologies have been identified as one of the top 10 Gartner strategic technology trends for 2019 (Panneta, 2018). So far, the main applications are in the gaming and entertainment sectors, but tourism, marketing, sports, education, and training have experimented substantial growth as well (Research and Markets, 2018). A study by Goldman Sachs (Heather Bellini, 2016) estimates that the size of the AR and VR markets will grow to $\$ 80$ billion by 2025 , similar to the size of the personal computer market in 2016. More recent reports estimate the size of the AR and VR market to grow to $\$ 94$ billion by 2023 (Research and Markets, 2018). Fifty-two out of the Fortune 500 companies are testing and deploying AR or VR solutions, and venture capital investment increased 230\% from 2016 to 2017 (Kaiser and Scatsky, 2017). Many companies from various sectors (e.g. aerospace, logistics, retail) are using AR and VR for education, training and productivity improvements. For example, Boeing (2018) reported up to $40 \%$ productivity improvements in electrical wiring installation tasks when using AR headmounted displays (HDMs) to support workers.

Despite the huge potential of AR and VR technologies, as with other digital technologies, their adoption in the Architecture, Engineering, and Construction (AEC) sectors is still very low. For example, the McKinsey Global Institute (Manyika et al., 2015) reported that the level of digitalization index for the construction industry was the lowest out of 22 industries. Results from a survey conducted by the authors for this study estimate a VR adoption factor in the UK construction sector of 2.5 out of 5; and of 1.5 out of 5 for AR (Davila Delgado et al., 2019a). In the factor scale, 5 represents full adoption, and 1 represents not used at all. These low levels of adoption are caused by a myriad of complex and interrelated factors that are very difficult to understand, and consequently, appropriate mitigating actions cannot be devised. This paper presents a systematic study of the factors that limit and drive the adoption of AR and VR technologies in the construction industry. The objectives of this study are:

(1) To identify, categorize, and rank the most relevant factors that limit and drive the adoption of $\mathrm{AR}$ and VR in the construction industry.

(2) To provide a clear and understandable explanation of the main factors that limit and drive adoption, which could be used as the basis to develop mitigating actions.

A mixed method approach, consisting of qualitative and quantitative data collection and analyses (see Figure 3), was employed to achieve the objectives above. Exploratory workshops - with experts from industry and academia - and quantitative data collection tools were used to identify and rank the factors. Statistical analyses were used to organize a large number of possibly correlated factors into a 
smaller number of uncorrelated factors. The uncorrelated factors are then explained in the context and the dynamics of the construction sector. The next section provides an overview of AR and VR technologies in the AEC context; next, the methodology used in this study is explained. Sections 4 and 5 present the qualitative and quantitative analyses, respectively. Sections 6 and 7 explain the limitations and drivers that have been identified. Section 8 discusses the findings presented, provides and comparison with other similar studies, and presents a roadmap to improve adoption. Lastly, conclusions are provided.

\section{Background}

\subsection{Virtual Reality, Augmented Reality, and Mixed Reality}

VR is the technology that enables the creation of entirely computer-generated environments that give the user the sensation of being completely immersed within a virtual environment. It provides a way to replace the perception of the surrounding world with a computer-generated artificial 3D environment. The virtual experience is provided usually through a head-mounted display (HMD), a device that provides a virtual experience to a single individual, but they are other room-sized systems that enable VR experiences for many individuals (e.g. DeFanti et al., 2009). VR can be used, for example, to train and test healthcare professionals by immersing them in virtual surgery rooms (Yiannakopoulou et al., 2015), in which they need to perform specific tasks without the need for expensive real-life facilities and human subjects. On the other hand, AR is the technology that enables to overlay digital information onto the real environment -in real-time and in the correct spatial position-to augment or enhance the real environment. In other words, AR enables digital objects and information to be overlaid either through an HMD or via a handheld device with a camera such as a smartphone or a tablet. For example, AR systems, reported in literature, enable users to view three dimensional virtual furniture on real environments (Young and Smith, 2016). These capabilities have migrated to commercial solutions such as AR mobile shopping apps, which allow to visualize 3D models of furniture and see how they would look and fit in various places around a house.

The term "Mixed Reality" was originally coined by Milgram (1994). It refers to the spectrum or "virtual continuum" in which different technologies exist based on how much of the real environment is displayed (see Figure 1). At one end of the spectrum is the real environment that everybody experiences daily. On the other end, resides the virtual environment, in which the real environment is completely replaced by virtual objects. Various technologies can be mapped within the Mixed Reality Spectrum. For example, VR is closer to the virtual environment end of the spectrum, in which the real environment is not displayed at all. While AR is closer to the other end, in which a combination of real environment objects and virtual environment objects are displayed. Other technologies can be mapped within this spectrum, for instance Augmented Virtuality (AV) refers to a virtual environment augmented with 
object from the real environment (e.g. Albert et al., 2014); or Tangible User Interfaces (TUI), which are physical objects from the real environment that enable a new way to interact with virtual objects (e.g. Skulmowski et al., 2016). Benford et al. (1998) presented a similar taxonomy to Milgram's one to explain the differences between AR and VR. In this case, the technologies are mapped in a fourquadrant space, in which two spectrums range from the physical world to the virtual world and from computer-generated data to physical data.

Milgram's definition of Mixed Reality is the most widely accepted in academia. However, recently the term Mixed Reality has been used by technology development companies to refer to a new distinct technology instead of a spectrum in which many technologies lie. This new definition has not been universally agreed upon, and many different definitions abound. In summary, it refers to a technology that, like AR, places virtual objects on a real environment, but that it also anchors them on the real world, and it enables interaction among physical and virtual objects. This new definition is very vague, lacks scientific rigor, and there are no reliable sources to support it. The new definition was primarily used as a marketing strategy to differentiate similar products. However, it seems that this new definition is losing traction, as now even the same technology development companies that introduced the new definition are using Milgram's definition as well (Bray and Zeller, 2018).

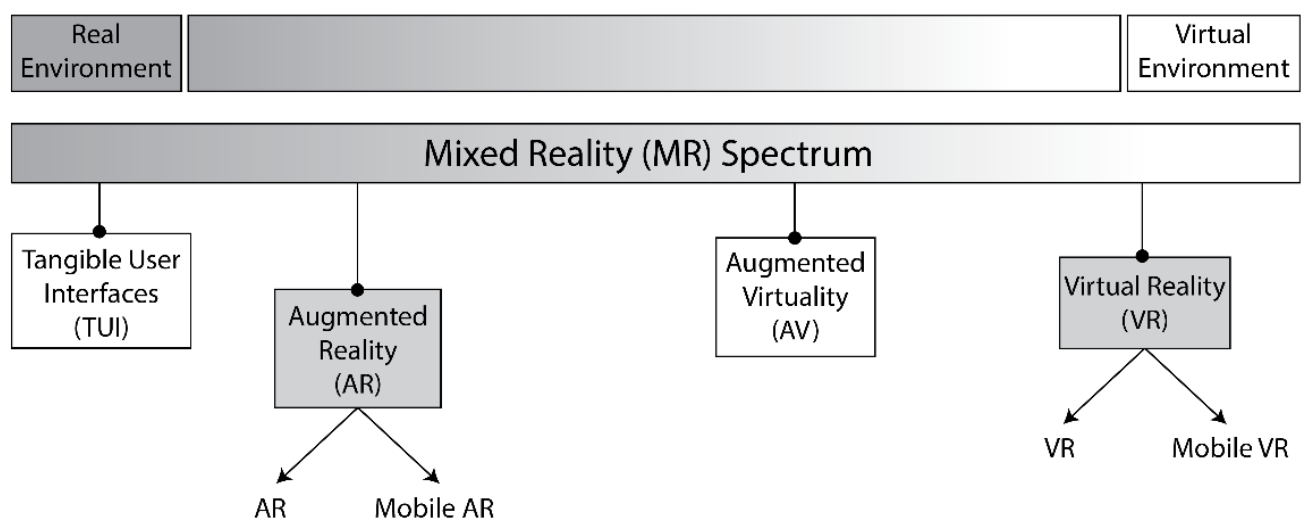

Figure 1. Diagram showing the Mixed Reality spectrum proposed by Milgram (1994) and AR and VR technologies within the spectrum.

Table 1 and Figure 2 presents the main types of AR and VR technologies. Both technologies have a mobile variant with fewer capabilities. AR and VR require HMDs and specialized controllers. The mobile version of AR does not require an HMD. Both mobile versions are less expensive and do not have high-processing requirements. Note that there are other types of AR and VR devices such as AR glasses, but they were not included here because they are not as developed, and there have not been applications related to the construction industry reported in literature. 
Table 1. Types of AR and VR technologies

\begin{tabular}{|c|c|c|c|c|}
\hline & VR & Mobile VR & $\mathbf{A R}$ & Mobile AR \\
\hline Processing & High & Low & Mid (built on HMD) & Low \\
\hline Tracking & $6 \mathrm{DOF}$ & $3 \mathrm{DOF}$ & $3 \mathrm{DOF}$ & $3 \mathrm{DOF}$ \\
\hline HMDs & $\begin{array}{l}\text { Yes, entirely } \\
\text { immersive (blacked- } \\
\text { out) HMD }\end{array}$ & $\begin{array}{l}\text { HMD adapter for } \\
\text { mobile phone }\end{array}$ & Yes, See-through HMD & No \\
\hline Sensors & $\begin{array}{l}\text { Infrared sensors on } \\
\text { HMD, } \\
\text { Stationary infrared } \\
\text { scanners }\end{array}$ & $\begin{array}{l}\text { Gyroscope, } \\
\text { Accelerometer, } \\
\text { Compass }\end{array}$ & $\begin{array}{l}\text { Depth camera, } \\
\text { Color and grayscale } \\
\text { cameras, } \\
\text { Infrared sensors }\end{array}$ & $\begin{array}{l}\text { Gyroscope, } \\
\text { Accelerometer, } \\
\text { Compass }\end{array}$ \\
\hline Controllers & $\begin{array}{l}\text { Infrared-tracked } \\
\text { controllers, } \\
\text { Infrared body trackers }\end{array}$ & $\begin{array}{l}\text { Non-tracked } \\
\text { controller }\end{array}$ & Tracks hand gestures & NA \\
\hline $\begin{array}{l}\text { Devices } \\
\text { (examples) }\end{array}$ & $\begin{array}{l}\text { Oculus Rift, } \\
\text { HTC Vive, } \\
\text { Sony PS VR, }\end{array}$ & $\begin{array}{l}\text { Any recent mobile } \\
\text { phone }\end{array}$ & $\begin{array}{l}\text { Microsoft HoloLens, } \\
\text { Magic Leap, } \\
\text { Meta } 2 \text { AR, } \\
\text { DAQRI }\end{array}$ & $\begin{array}{l}\text { Any recent } \\
\text { mobile phone or } \\
\text { tablet }\end{array}$ \\
\hline \multicolumn{5}{|c|}{$\begin{array}{l}\text { Data in this table is based on: } \\
\begin{aligned}- & \text { https://en.wikipedia.org/wiki/Comparison_of_virtual_reality_headsets } \\
- & \text { https://en.wikipedia.org/wiki/Optical_head-mounted_display }\end{aligned}\end{array}$} \\
\hline
\end{tabular}

\begin{tabular}{|c|c|c|c|c|}
\hline & VR & Mobile VR & $A R$ & Mobile AR \\
\hline Device & & & & \\
\hline
\end{tabular}

All images are available under the Creative Commons CCO 1.0 Universal Public Domain Dedication unless otherwise indicated.

(a) https://commons.wikimedia.org/wiki/File:Oculus-Rift-CV1-Headset-Front_with_transparent_background.png

(b) https://commons.wikimedia.org/wiki/File:Google-Cardboard.jpg

(c) https://commons.wikimedia.org/wiki/File:Meta_2.jpg. This file is licensed under the Creative Commons Attribution-Share Alike 4.0 International license.

(d) https://www.pexels.com/photo/3d-augmented-reality-medical-science-315437/. Creative Commons CCO 1.0 Universal Public Domain Dedication.

Figure 2. Examples of devices used: VR, mobile VR, AR, and mobile AR.

\subsection{AR and VR in Architecture, Engineering and Construction}

AR and VR technologies are of utmost importance for the AEC industry as a whole as the built environment is intrinsically linked to 3D space, and AEC professionals rely heavily on imagery for communication. In the UK, the Data for the Public Good report (National Infrastructure Commission, 2017) considered AR and VR as key new technologies to increase the productivity on infrastructure delivery, maintenance, and support decision-making. Similarly, in the USA, the government's information technology initiatives include an AR and VR initiative. In 2017, the emerging Citizen Technology Office launched the Federal Virtual/Augmented Reality program to coordinate the collaboration for the research and refinement of AR and VR business cases and pilot programs (GSA, 
2017). The US federal agencies expect that AR and VR technologies can potentially expand and improve their services in a wide range of applications from post-traumatic social disorder treatment, to educating farmers on the installation of solar panels, and disaster management preparedness and response (GSA, 2017).

AR is considered as an essential technology to improve construction projects (Woyke, 2016). Research on both AR and VR has been carried out for many decades, but recently the field has resurged driven by the development of new, more capable HMDs. Nevertheless, adoption in the AEC sectors of these technologies remains very low and circumscribed to very specific use cases. For example, client engagement using VR. The Manufacturing Technology Centre (MTC), in collaboration with i3P, a consortium of large construction companies and infrastructure providers, carried out an exploratory study into the maturity and applicability of AR and VR in construction companies in 2017 (MTC, 2017). The report reveals that only $37 \%$ of construction companies have some experience with AR and VR. This result is aligned with the authors' own research, which estimates that only $32.4 \%$ of construction companies in the UK have used AR or VR at some capacity (Davila Delgado et al., 2019a).

There are many varied use cases of AR for architecture, engineering and construction. However the main use case reported in literature is to assist with construction tasks, assembly operations and construction of pre-fabricated construction elements (e.g. Webster et al., 1996). Ahn et al. (2019) presented a projection-based AR approach for visualizing vital information within a user's field of view during panel manufacturing for construction. The authors state that their approach will improve the quality of the final manufactured products by reducing the offset distances and ensuring that are within the tolerance levels. Fazel and Izadi (2018) presented an AR system that supports construction workers to construct complex double-curved brick walls. The presented system uses a marker-based approach, in which a camera tracks two markers, one located on the floor and another one on the worker's HMD. The AR system computes the correct location of the wall to be constructed using the relative position and orientation of both markers. Then, visual guides are displayed on the worker's HMD indicating the correct position and orientation of the bricks required for constructing the wall. Chalhoub and Ayer (2018) presented an AR system that supports workers to install electrical installations at the correct positions. Using AR, a 3D model of electrical conduits is overlaid at the correct position in the room, obviating the need for 2D drawings. Deshpande and Kim (2018) investigated the effects of AR to support assembly tasks. The authors developed an AR system that provided visual guides to assemble furniture pieces and found indications that AR guidelines can improve the understanding of spatial relationships among components.

Other AR use cases include: (1) see-through opaque surfaces (e.g. walls, floor, road surfaces, etc.) to visualize construction elements and infrastructure assets (e.g. gas, water, or electricity underground cables) (Schall et al., 2009). (2) Support finding assets (e.g. power distribution boxes, pipe mains, etc.) 
in complex sites (Neges and Koch, 2016), (3) Support design reviews (Dong et al., 2013; Schubert et al., 2015). For example, Lin et al. (2019) presented an AR system that visualizes the results of computer fluid dynamics simulations of indoor thermal environments on mobile devices. (4) Support collaborative design and the development of layouts (Nee et al., 2012). (5) Improve the information retrieval process during construction (Behzadi, 2016). Chu et al. (2018) investigated how AR can be used to improve information retrieval from BIM models using markers. (6) Enhance collaboration and facilitate remote support (Billinghurst and Kato, 2002). (7) Query physical objects by querying aligned but hidden model elements (Seo and Lee, 2013) (8) Verify whether new equipment will fit and for clash detection (Friedrich, Jahn and Schmidt, 2002). (9) Improve building site monitoring and inspections (Golparvar-Fard, Peña-Mora and Savarese, 2009). For example, Zhou et al. (2017) presented an AR approach to support the inspection of segment displacement during tunneling construction. The approach enables to overlay a quality control baseline model onto the real segment and measure the differences. (10) Support asset and facility management (Schall, Mendez and Schmalstieg, 2008; Palmarini et al., 2018). For instance, Baek et al. (2019) presented an AR approach for facility management that presents location-specific data in AR using image-based indoor localization. The marker-less approach estimates the user's indoor position and orientation by comparing the user's perspective with a predefined BIM model. Neges et al. (2017) presented an AR framework that digitally supports facility maintenance operators when navigating indoors. The framework combines a step counter device and visual live video feed to provide accurate indoor navigation support. (11) Support education and training (e.g. Eiris Pereira et al., 2019). Turkan et al. (2017) presented an AR system to teach structural analysis and to help students understanding the behavior of structural elements in a three-dimensional context. The AR system enables students to define simple structural systems and interactively change the loads and observe the reaction with the instant feedback provided by the AR interface.

The application of VR in the AEC sector is arguably more mature than that of AR. The textbook by Whyte and Nikolic (2018), reviews the practical applications of VR in the design, construction, and management of the built environment. The main use cases include: (i) support with design reviews (Dunston et al., 2011; Aromaa and Väänänen, 2016; Berg and Vance, 2016). For example, Boton (2018) proposed a method to support constructability analysis meetings using VR environments. The method enables to export BIM-based construction simulations into a VR application for immersive visualization. Wolfartsberger (2019) presented a VR system for engineering design review, in which faults in designs were easier to identify, and the review process was carried out faster compared with traditional review processes. (ii) Support with immersive design and drafting (Whyte et al., 2000; Roach and Demirkiran, 2017). For instance, Lin et al. (2018) developed a VR approach to support the design of healthcare facilities by improving the communication between the design teams and healthcare stakeholders. Du et al. (2018b) presented an approach that enables real-time synchronization of BIM 
data with VR applications. The approach enables to update a BIM model based on the changes made in VR application automatically and simultaneously, e.g. changing object dimensions, changing object locations and changing object types. (iii) Facilitate the creation of more useful simulations and testing of design solutions. (Mujber, Szecsi and Hashmi, 2004; Rekapalli and Martinez, 2011). Motamedi et al. (2017) presented an approach to test the effectiveness of signages of Japanese subway stations on VR environments. Most notably, Ergan et al. (2019) used a set of biometric sensors, and physiological metrics such as skin conductance, brain activity, and heart rate to provide an indication of the levels of stress and anxiety users experienced in VR environments. The authors claim that their approach provides a systematic way for architectural design firms to get accurate user feedback before the design is finalized. (iv) Improve education and training (Boud et al., 1999; Zhao and Lucas, 2015). Fogarty et al. (2018) investigated how VR can be used to improve the student's spatial understanding of complex spaces. (v) Improve health and safety. For example, Albert et al. (2014) presented a VR method for hazard identification in construction sites. Lovreglio et al. (2018) developed a VR solution to assess the level of preparedness for building evacuations during earthquakes. Shi et al. (2019) used VR to assess distinct types reinforced learning methods on the behavior of construction workers associated with fall risks. Dris et al. (2019) proposed a VR approach that supports risk identification and improves the data exchange between BIM models and VR applications. Lastly, (vi) improving stakeholders' engagement and communication (Annetta et al., 2009; Du, Shi, et al., 2018; Hassan, Taib and Rahman, 2018). Pratama and Dossick (2019) conducted a study with AEC companies and identified that majority of companies use VR for generating immersive building walkthroughs.

Note that there are no studies reported in literature that analyze the limiting and driving factors influencing the adoption of AR and VR in construction. Most of the studies addressing adoption limitations focus only on technical aspects e.g. the work by Behzadan et al. (2015) and Palmarini et al. (2018); or on specific use-cases e.g. the work of Li et al. (2018) on construction safety. Nevertheless, section 8.1 presents a detailed comparison of the findings of this study with the studies above and with other AR and VR adoption studies carried out in other fields (Tourism, Healthcare, and Education).

\section{Research Methodology}

A mixed research method, as presented by Creswell (2017), was used for this study. It combines qualitative data collection and analysis and quantitative data collection and analysis. This type of mixed research method has been proved to be a powerful tool to investigate complex processes and systems in other areas, such as the healthcare sector (Fetters, Curry and Creswell, 2013). For this reason, they were selected to be used in this study. These types of mixed methods are very useful in identifying underlying factors in complex systems by supporting and guiding the quantitative data collection and analysis with qualitative research activities. The combination of both qualitative and quantitative analyses helps to explain, categorize and generalize findings (Fetters, Curry and Creswell, 2013). Figure 
3 presents a diagram of the research methodology used. The first step, qualitative analysis, was to conduct four exploratory workshops with experts in the field, from industry and academia, in which a series of focus group discussions (FGDs) were conducted (Table 2). Findings from the FGDs were compiled into two lists of factors that limit and drive adoption. In the second step, quantitative analysis, the results from the previous step were used to develop a quantitative data collection instrument, i.e. questionnaire. The questionnaire was administered to specialists and academics with expertise in AR and VR based in the UK; the sampling method used, and the distribution of the participants are detailed in section 5.1. Using the results of the questionnaire, the identified factors were ranked and categorized using principal component analysis (PCA). A reliability analysis was carried out on the questionnaire results to validate the internal consistency of the results, and multivariate analysis of covariance was carried out to test whether there were significant statistical differences in the responses that could be attributed to the varied profiles of the respondents. Lastly, using the results of the qualitative and the quantitative analysis, relevant insights into the factors limiting and driving the adoption of AR and VR in the construction industry were drawn and explained.

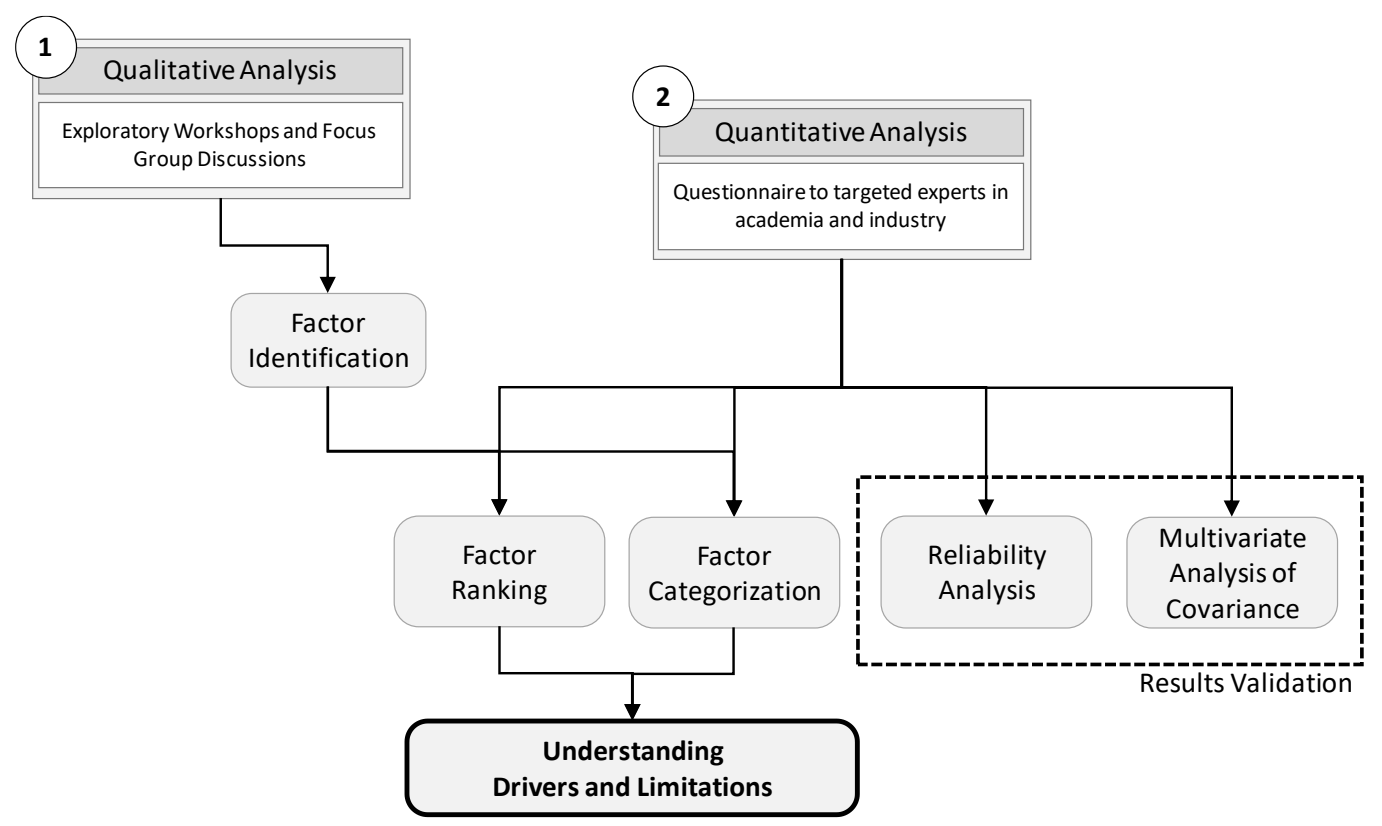

Figure 3. Diagram of the mixed research methodology used in this study.

\section{Qualitative sampling and analysis}

The main activities conducted in the qualitative part of the study were four exploratory workshops, in which two FGDs were carried out at each workshop. In total, 8 FGDs were held with durations between 30 and 45 minutes. Fifty-four experts from 36 organizations, companies, and academic institutions based in the UK participated in workshops. The intention was to assemble multidisciplinary expert groups with varied expertise; therefore, experts from academia, construction companies, design offices, engineering consultancies, and technology development companies were invited to participate. The 
invited experts had to be working on AR and VR, have more than 3-year experience, and working on a company from the categories mentioned above. The size of the groups was capped at $\sim 15$ experts so that the discussions could be managed more easily, and all the participants could have the opportunity to participate. All the participants were different for each workshop. An overview of the participants and the FGDs are presented in Table 2.

The FGDs were used to collect the opinion of experts on the field regarding factors that limit and drive the adoption of AR and VR in the construction industry. FGDs are very effective tools for qualitative and exploratory analysis as they allow the participants to build on arguments from the other participants (Kvale, 1994). This is not the case with individual interviews, in which important factors could be missed in the study. A thematic analysis based on an example from literature was used for the qualitative part of the study, which includes: (1) data familiarization, (2) data coding and segmentation, (3) development of themes, and (4) grouping of related themes. Each FGD consisted of two activities: a factor identification activity, in which small groups of participants identified factors; and a group discussion session, in which all the identified factors were discussed among all the participants. In all the activities, a member of the research team was present to guide the activities. The FGDs were audiorecorded and notes were taken by hand. All the data generated in the FGDs were compiled and categorized into tables. Tables 3 and 4 present samples of the type of data compiled from the FGDs. This data was used to develop a quantitative data collection instrument, which is explained in the next section.

Table 2. Overview of the workshops and focus group discussions

\begin{tabular}{|c|c|c|c|c|c|}
\hline Workshops & Participants & $\begin{array}{c}\text { No of } \\
\text { experts }\end{array}$ & FGD & Topics & Duration \\
\hline W1 & $\begin{array}{l}>4 \text { University researchers } \\
>1 \text { Standards organization } \\
>4 \text { Contractors } \\
>1 \text { Architect } \\
>2 \text { Engineering consultants } \\
>2 \text { Technology developers }\end{array}$ & 14 & $\begin{array}{l}1.1 \\
1.2\end{array}$ & $\begin{array}{l}\text { Identify limitations } \\
\text { - Identify drivers }\end{array}$ & $\begin{array}{l}45 \mathrm{~min} \\
40 \mathrm{~min}\end{array}$ \\
\hline W2 & $\begin{array}{l}>4 \text { University researchers } \\
>4 \text { Contractors } \\
>3 \text { Engineering consultants } \\
>2 \text { Technology developers }\end{array}$ & 13 & $\begin{array}{l}2.1 \\
2.2\end{array}$ & $\begin{array}{l}\text { Identify limitations } \\
\text { - Identify drivers }\end{array}$ & $\begin{array}{l}45 \mathrm{~min} \\
40 \mathrm{~min}\end{array}$ \\
\hline W3 & $\begin{array}{l}>5 \text { University researchers } \\
>4 \text { Contractors } \\
>1 \text { Architect } \\
>3 \text { Engineering consultants } \\
>3 \text { Technology developers }\end{array}$ & 16 & 3.1 & $\begin{array}{l}\text { Rank and qualify } \\
\text { limitations } \\
\text { - Rank and qualify drivers }\end{array}$ & $\begin{array}{l}45 \mathrm{~min} \\
45 \mathrm{~min}\end{array}$ \\
\hline W4 & $\begin{array}{l}\text { > } 3 \text { University researchers } \\
\text { > } 1 \text { Infrastructure manager } \\
\text { > } 3 \text { Contractors } \\
\text { > } 1 \text { Architect } \\
\text { > } 3 \text { Engineering consultants }\end{array}$ & 11 & $\begin{array}{l}4.1 \\
4.2\end{array}$ & $\begin{array}{l}\text { Rank and qualify } \\
\text { limitations } \\
\text { - Rank and qualify drivers }\end{array}$ & $40 \mathrm{~min}$ \\
\hline
\end{tabular}


Table 3. A sample of the findings to identify factors that limit the adoption of AR and VR in construction.

\begin{tabular}{lll}
\hline Social Limitations & Technical Limitations & Economic Limitations \\
\hline $\begin{array}{l}\text { Aversion to change } \\
\text { Lack of trained workforce } \\
\text { Fragmented industry }\end{array}$ & Battery limitations & Expensive hardware \\
Clunky user interface & Awkward VR sensors & Expensive training \\
Job security issues & Lack of information exchange & $\begin{array}{l}\text { Predominance of SME's in the } \\
\text { sector }\end{array}$ \\
$\begin{array}{l}\text { Branding issues } \\
\text { Issues with data privacy and } \\
\text { ownership }\end{array}$ & Low Field of View (FOV) & High-risk industry \\
$\begin{array}{l}\text { Health and safety issues } \\
\text { Isolation caused by HMDs } \\
\text { Mobility issues }\end{array}$ & Accuracy issues & Low profits \\
\hline
\end{tabular}

Table 4. A sample of the findings to identify factors that drive the adoption of AR and VR in construction.

\begin{tabular}{|c|c|c|c|c|}
\hline Designers & Contractors & Managers & Owners & End-users \\
\hline $\begin{array}{l}\text { Timely feedback. } \\
\text { Engagement } \\
\text { feedback } \\
\text { Better } \\
\text { understanding of } \\
\text { cross-discipline } \\
\text { interaction } \\
\text { Efficient design } \\
\text { making } \\
\text { Visual quick } \\
\text { understanding of a } \\
\text { complete program }\end{array}$ & $\begin{array}{l}\text { Better site } \\
\text { planning. } \\
\text { Contextual } \\
\text { understanding. } \\
\text { Reduce overall } \\
\text { cost. } \\
\text { Better cost and } \\
\text { time performance. } \\
\text { Risk reduction. } \\
\text { Re-skilling }\end{array}$ & $\begin{array}{l}\text { Impact assessment } \\
\text { Cost reduction } \\
\text { More efficient } \\
\text { space planning } \\
\text { Informed decision- } \\
\text { making support }\end{array}$ & $\begin{array}{l}\text { Reduce risk and } \\
\text { less cost } \\
\text { Improve quality } \\
\text { Simulations give } \\
\text { better } \\
\text { understanding of } \\
\text { the design and built } \\
\text { environment. } \\
\text { Reduce cost on } \\
\text { project delivery }\end{array}$ & $\begin{array}{l}\text { Understanding } \\
\text { social impact of } \\
\text { governmental new } \\
\text { construction } \\
\text { developments. } \\
\text { Inclusivity. } \\
\text { Improve buyer } \\
\text { experience } \\
\text { (clarity). } \\
\text { Issues resolved } \\
\text { more easily } \\
\text { Social engagement } \\
\text { and clarity for } \\
\text { government } \\
\text { planning. } \\
\text { Enhance user } \\
\text { experience. }\end{array}$ \\
\hline
\end{tabular}

\section{Quantitative sampling and analysis}

Based on the findings of the FGDs and the qualitative analysis, 21 limiting factors and 21 driving factors for the adoption of AR and VR in the construction industry were defined, as shown in Tables 6 and 7, respectively. A questionnaire was developed to validate and quantify the importance of both sets of factors. A 1 to 5 Likert scale was used in the questionnaire to codify the responses, in which 1 corresponds to the lowest importance and 5 to the highest importance. The respondents were asked to assign an importance value to each of the limiting and driving factors. The questionnaire was pilottested by 6 experts ( 4 from industry and 2 from academia) to ensure the clarity of the questions and the structure and logic of the questionnaire. 


\subsection{Respondents composition}

Experts from academia, construction companies, engineering consultancies, design firms, and technology development companies, focused on AR and VR and based in the UK, were approached to participate. A combination of convenience and stratified sampling methods was used to target potential participants. Stratification was carried out by dividing the potential participants into categories based on the type and size of their organizations. Between 3 to 5 experts from the following eight categories were targeted to participate: (i) top construction companies by revenue, (ii) small and medium construction companies, (iii) top engineering consultancy companies by number of employees, (iv) small and medium engineering consultancy companies, (v) top design firms by number of employees, (vi) small and medium design firms, (vii) technology development companies, and (viii) academia. Within the defined categories, the experts that were readily available to participate were selected (convenience sampling), instead of selecting experts randomly.

In total, 45 experts were contacted, and 34 completed questionnaires were received, which represents a $75.5 \%$ response rate. The distribution of the respondents is as follows (see Table 5): $11.8 \%$ are researchers from academic institutions, $20.6 \%$ work in construction companies, 32.4\% work in engineering consultancies, and $17.6 \%$ work in design or architecture offices and in technology development companies. Based on the participants' responses, an expertise level factor was developed to provide an indication of the relevant experience of the respondents. This factor is the average of the following self-declared attributes of the participants: (1) years of professional experience, (2) years of experience using AR\&VR, (3) level of implementation complexity in VR, and (4) level of implementation complexity in AR. The distribution of the expertise level factor of the participants is presented in Table 5, in which more than $75 \%$ of the respondents identify themselves as advanced or experts in the field.

Table 5. Overview of the respondents.

\begin{tabular}{llll}
\hline Variables & Groups & Frequency & Percentage \\
\hline Role & Academia & 4 & $11.8 \%$ \\
& Construction & 8 & $23.5 \%$ \\
& Engineering consultancy & 11 & $32.4 \%$ \\
& Design & 6 & $17.6 \%$ \\
& Technology development & 5 & $14.7 \%$ \\
Expertise level & & \\
& & 0 & \\
& Novice (0-0.9) & 1 & $2.9 \%$ \\
& Beginner (1-1.9) & 7 & $20.6 \%$ \\
& Intermediate (2-2.9) & 17 & $50.0 \%$ \\
& Advanced (3-3.9) & 9 & $26.5 \%$ \\
\hline
\end{tabular}




\subsection{Reliability analysis and multivariate analysis of covariance}

A reliability analysis was conducted to test the internal consistency of the factors included in the questionnaire. Two metrics were used for the reliability analysis, i.e., Cronbach's Alpha, which is a correlation estimate for randomly equivalent measures; and Gutman's lambda-2, which estimates correlation for parallel measures. The Cronbach's Alpha and Gutman's lambda-2 for the limiting factors are 0.749 and 0.791 (Table 6), and for the driving factors are 0.669 and 0.729 (Table 7). The obtained metrics, for both limiting and driving factors, indicate an acceptable internal consistency of the collected data (Nunnally and Bernstein, 1994). A multivariate analysis of covariance was conducted as well to identify whether the different roles of the participants had a significant statistical influence in their responses while controlling for their difference in expertise level. The Pillai's Trace test statistic was used for the multivariate analysis of covariance, which resulted higher than 0.05 for both the limiting factors (0.478) and the driving factors (0.514) (Table 6 and 7 respectively). This indicates that the different roles do not have a significant effect when controlling for the difference in expertise level (Morrison, 2005).

\subsection{Results}

Table 6 and Table 7 present the limiting and driving factors ranked according to the mean value of all the responses, respectively. The median, standard deviation (SD), and skewness are presented as well. The median is presented because it is not affected by outliers, so that very disparate answers do not affect the overall results. The SD provides an indication of how dissimilar the answers are. In this case, it indicates how dissimilar the answers are among respondents. Low SD indicates that respondents agree on the importance of the factors, and high SD indicates disagreement. The highest SD for this study is 1.4, which can be considered a low value and may indicate a good agreement among respondents. Skewness measures the degree and direction of asymmetry. A negative skewness indicates that the mean is less than the median and that the distribution of responses is concentrated in high values. A large positive skewness indicates that the distribution of the responses is concentrated in low values; and a skewness equal to zero indicates a normal distribution. Figures 2 and 3 present the distribution of the importance of the limiting and driving factors using Letter-Value plots (Hofmann, Wickham and Kafadar, 2017). Letter-Value plots are a variation of box plots that show more quantiles and provide more information about tail behavior. Letter-Value plots provide a non-parametric representation of a distribution in which all features correspond to actual observations. Using Letter-Value plots is possible to visualize smaller differences among distributions that box plots cannot present. The vertical scales in Figures 2 and 3 indicate the importance of each factor. Different shades of color have been used to indicate the varying medians of each factor. Darker shades indicate higher medians, while lighter shades indicate lower medians. 
The highest-ranked limiting factor is (L11) Expensive hardware and training. It has the lowest SD and a negative skewness, which indicates that for most of the respondents this factor is the most important. The lowest-ranked factor is (L18) It is seen as a cause for job insecurity, which has one of the highest SD and a positive skewness. This indicates variation of opinions between respondents and an overall low importance. The overall standard deviation for limiting factors is 1.18 , which indicates that the responses between respondents varied on average less than 1.2 points in the Likert scale. Looking at Figure 4 is possible to identify that six limiting factors (L2, L8, L9, L11, L16, and L21) are regarded as the most important with a median of 4 . Limiting factor L4 has the second level of importance (median of 3.5), and the rest have medium importance (median of 3), with the exception of limiting factor L18, which is the less important (median of 2.5).

The highest-ranked driving factor is (D11) A way to provide new and better services. It has an average $\mathrm{SD}$ and a large negative skewness, which indicates that for most of the respondents this factor is the most important. The lowest-ranked factor is (D4) to be part of the trend, which has one of the highest SD and a large positive skewness. This indicates a variation of opinions between respondents and overall low importance. Similarly, to the limiting factors, the overall standard deviation for driving factors is 1.183 . Figure 5 presents D8 and D1 1 as the most important driving factors with a median of 4.5. Thirteen driving factors are considered as very important with a median of 4 and seven as somewhat important with medians of 3.5 and 3.

Table 6. Ranked list of identified limitations.

\begin{tabular}{cllcccc}
\hline Rank & Label & Limitations & Mean & Median & Std. & Skewness \\
\hline 1 & L11 & Expensive hardware and training & 3.85 & 4.0 & 0.958 & -0.350 \\
2 & L2 & Specialized high processing equipment requirements & 3.74 & 4.0 & 0.931 & -0.145 \\
3 & L16 & Aversion to adopt new technologies & 3.74 & 4.0 & 1.163 & -1.043 \\
4 & L21 & Skill shortage and difficulty to access skilled graduates & 3.68 & 4.0 & 1.224 & -0.489 \\
5 & L9 & Lack of standards for data exchange & 3.62 & 4.0 & 1.206 & -0.624 \\
6 & L8 & Limited size of 3D models to be displayed & 3.59 & 4.0 & 1.184 & -0.515 \\
7 & L4 & Lack of multi-user capabilities & 3.44 & 3.5 & 1.050 & -0.503 \\
8 & L15 & Lack of time to explore immersive technologies & 3.35 & 3.0 & 1.252 & -0.136 \\
9 & L20 & Difficulties to access expert knowledge & 3.29 & 3.0 & 1.169 & -0.376 \\
10 & L19 & Fragmented industry & 3.26 & 3.0 & 1.163 & -0.308 \\
11 & L13 & Limited access to finance & 3.24 & 3.0 & 1.350 & -0.221 \\
12 & L5 & Uncomfortable and heavy HMDs & 3.18 & 3.0 & 1.167 & 0.000 \\
13 & L1 & Power and battery limitations & 3.12 & 3.0 & 1.320 & -0.313 \\
14 & L7 & Low resolution displays & 3.12 & 3.0 & 1.122 & 0.166 \\
15 & L12 & Lack of client's interest & 3.03 & 3.0 & 1.291 & 0.032 \\
16 & L17 & Branding problems and inaccurate public perception & 3.03 & 3.0 & 1.243 & -0.159 \\
17 & L3 & Large space requirements & 3.00 & 3.0 & 0.921 & 0.000 \\
18 & L14 & Lack of market knowledge & 2.94 & 3.0 & 1.301 & 0.115 \\
19 & L6 & Narrow field of view & 2.91 & 3.0 & 1.240 & -0.026 \\
20 & L10 & Issues with data security and ownership & 2.82 & 3.0 & 1.403 & 0.123
\end{tabular}


Overall Std Dev $=1.18$.

Overall Cronbach's Alpha $=0.749$; Overall Gutman's lambda-2 $=0.791$.

Pillai's Trace Significance $=0.478$.

Table 7. Ranked list of identified factors driving adoption.

\begin{tabular}{cclcccc}
\hline Rank & Label & Factors driving adoption & Mean & Median & Std. & Skewness \\
\hline 1 & D11 & A way to provide new and better services. & 4.18 & 4.5 & 1.167 & -1.823 \\
2 & D19 & AR and VR improves project understanding. & 4.15 & 4.0 & 0.702 & -0.213 \\
3 & D12 & Increasing R\&D investment in the construction sector. & 4.12 & 4.0 & 1.038 & -1.804 \\
4 & D8 & AR and VR reduces overall risks. & 4.06 & 4.5 & 1.205 & -1.224 \\
5 & D15 & The need to increase labor productivity in the sector. & 4.03 & 4.0 & 1.029 & -1.124 \\
6 & D20 & AR and VR improves collaboration between parties. & 3.91 & 4.0 & 0.866 & -0.418 \\
7 & D5 & Organization's need to be more efficient and productive. & 3.88 & 4.0 & 1.066 & -1.027 \\
8 & D17 & Adequate marketing of AR and VR technologies. & 3.74 & 4.0 & 1.333 & -0.705 \\
9 & D16 & Government incentives. & 3.71 & 4.0 & 1.338 & -0.874 \\
10 & D10 & Enables market expansion. & 3.68 & 4.0 & 1.387 & -0.752 \\
11 & D7 & Improves the reputation of the organization. & 3.65 & 4.0 & 1.300 & -0.608 \\
12 & D21 & AR and VR contributes to better project delivery. & 3.53 & 3.0 & 1.237 & -0.123 \\
13 & D9 & Improves the organization's work culture. & 3.47 & 4.0 & 1.376 & -0.491 \\
14 & D6 & Fostering research curiosity of the employees. & 3.41 & 3.5 & 1.328 & -0.416 \\
15 & D13 & Decrease in construction budgets will drive adoption. & 3.41 & 4.0 & 1.158 & -0.395 \\
16 & D14 & Difficulties to access labor will drive adoption. & 3.41 & 4.0 & 1.158 & -0.395 \\
17 & D1 & Client requires the use of AR\&VR. & 3.38 & 3.5 & 1.577 & -0.238 \\
18 & D3 & Obtaining a differentiating advantage in the market. & 3.26 & 3.0 & 1.263 & -0.055 \\
19 & D18 & AR and VR will reduce overall spending in projects. & 3.09 & 3.0 & 1.190 & 0.050 \\
20 & D2 & Strategic decision from top management. & 3.03 & 3.0 & 1.381 & -0.056 \\
21 & D4 & To be part of the trend. & 2.62 & 3.0 & 1.326 & 0.352 \\
\hline Or & & & &
\end{tabular}

Overall Std Dev $=1.183$.

Overall Cronbach's Alpha $=0.669$; Overall Gutman's lambda-2 $=0.729$.

Pillai's Trace Significance $=0.514$. 


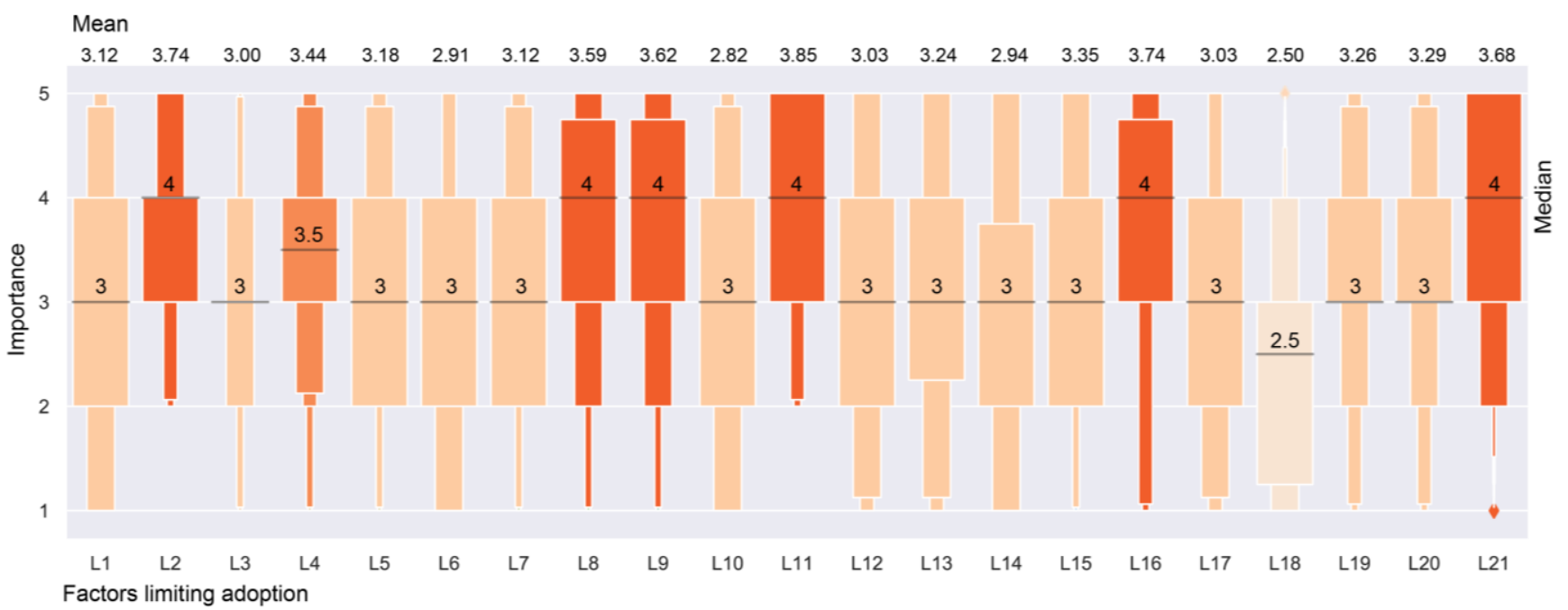

Figure 4. Overview of the importance of the factors that limit adoption. Factor L11 (Expensive hardware and training is the highest ranked factor. Darker shades indicate higher importance, while lighter shades indicate lower importance.

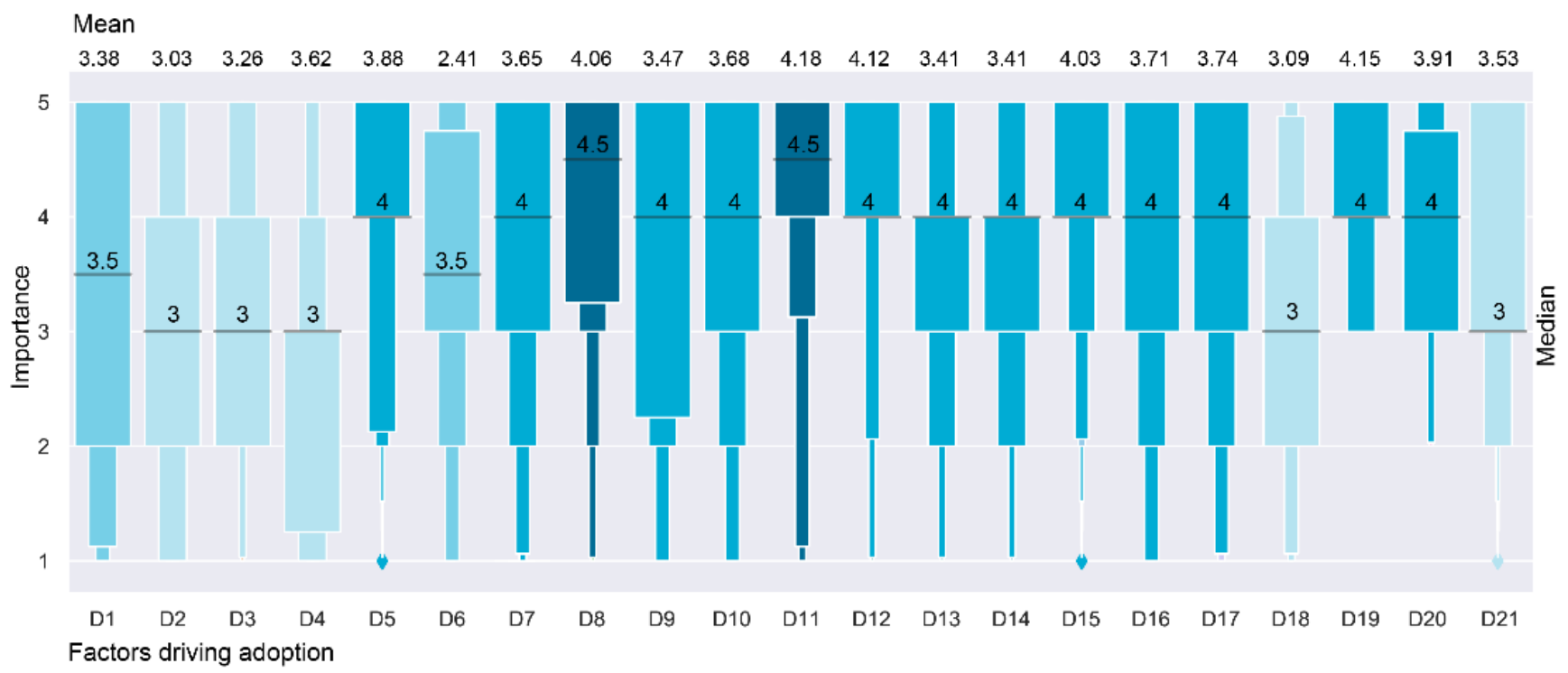

Figure 5. Overview of the importance of the factors that drive adoption. Factor D11 (A way to provide new and better services) is the highest ranked factor. Darker shades indicate higher importance, while lighter shades indicate lower importance.

\subsection{Principal component analysis}

Principal Component Analysis (PCA) is a data reduction tool that enables to represent a complex scenario with a large number of correlated variables with fewer uncorrelated variables. In this case, PCA was carried out to identify a smaller set of underlying factors from the previously identified limiting and driving factors. The intention of this analysis is to identify underlying correlations among the identified factors and group them into a smaller set of components. In other words, the intention is to create groups of similar and related factors. Reducing the 42 identified factors into a more 
manageable number of grouped factors facilitates understanding and contributes to devising actions to drive up adoption. PCA was used to capture as much information in the original factors based on the correlations among them. Tables 8 and 9 present the results of the PCA conducted on the limiting and driving factors, respectively. Four components, or groups of factors, were extracted from the limiting factors and four from the driving factors. Tables 8 and 9 present (1) the percentage of variance, an indication of how much certain component and its grouped factors contribute to representing the complex scenario that the factors described. A higher percentage indicates that the component represents better the correlation between factors. (2) The defining factor loading, an indication of how much a factor belongs to a certain component, and (3) the factors communalities, which are estimates of the variance accounted by the factors. Communalities are a metric between 0 and 1 , with high values indicating that the extracted components represent the variables well. Note that the factors within each component are ordered based on their defining factor loading and not their importance.

Table 8 shows that the four components account for more than $75 \%$ of the variance in the responses for the limiting factors. In other words, these four categories represent the underlying key points of $75 \%$ of the most important limiting factors. Table 9 shows that the four components account for more than $77 \%$ of the variance for the driving factors. The factor loadings and communalities from the limiting and the driving factors indicate that the extracted components represent well all the underlying factors. The components were interpreted into categories and named based on the assigned factors. For the limiting factors, the four categories defined are: (1) "Immature technologies", (2) "Non-technical issues", (3) "Special requirements for implementation", and (4) "Sector structure and client-contractor dynamics". For the driving factors the four categories defined are: (i) "Improving performance in projects", (ii) "Improving the companies' image", (iii) “Improving companies' overall performance", and (iv) "Bolstering research and development". Note that the categories are ordered based on the percentage of variance that they represent. These eight categories represent the main factors limiting and driving the adoption of AR and VR in the construction industry and are discussed in the next sections.

Table 8. Four components extracted that group the factors limiting adoption

\begin{tabular}{clccc}
\hline Label & Categories and factors & $\begin{array}{c}\text { \% of variance } \\
\left(\% \sigma^{2}\right)\end{array}$ & $\begin{array}{c}\text { Factor loading } \\
(f)\end{array}$ & $\begin{array}{c}\text { Communalities } \\
(\text { extraction })\end{array}$ \\
\hline CL1 & Immature technologies & $\mathbf{2 4 . 9 6 \%}$ & & \\
\hline L8 & Limited size of 3D models to be displayed & & 0.729 & 0.720 \\
L5 & Uncomfortable and heavy HMDs & 0.688 & 0.739 \\
L6 & Narrow field of view & & 0.668 & 0.741 \\
L7 & Low resolution displays & & 0.644 & 0.808 \\
L1 & Power and battery limitations & 0.628 & 0.780 \\
L21 & Skill shortage and difficulty to access skilled graduates & & 0.599 & 0.652 \\
& & & & \\
\hline CL2 & Non-technical issues & $\mathbf{2 2 . 7 8 \%}$ & & 0.746 \\
\hline L14 & Lack of market knowledge & & 0.756 & 0.825 \\
L10 & Issues with data security and ownership & & 0.737 & 0.855 \\
L17 & Branding problems and inaccurate public perception & & 0.729 &
\end{tabular}


L15 Lack of time to explore immersive technologies

L18 It is seen as a cause for job insecurity

\begin{tabular}{clll}
\hline CL3 & Special requirements for implementation & $\mathbf{1 9 . 7 6 \%}$ & \\
\hline L9 & Lack of standards for data exchange & 0.688 & 0.773 \\
L11 & Expensive hardware and training & 0.625 & 0.764 \\
L2 & Specialized high processing equipment requirements & 0.609 & 0.861 \\
L3 & Large space requirements & 0.605 & 0.759 \\
L4 & Lack of multi-user capabilities & 0.512 & 0.771
\end{tabular}

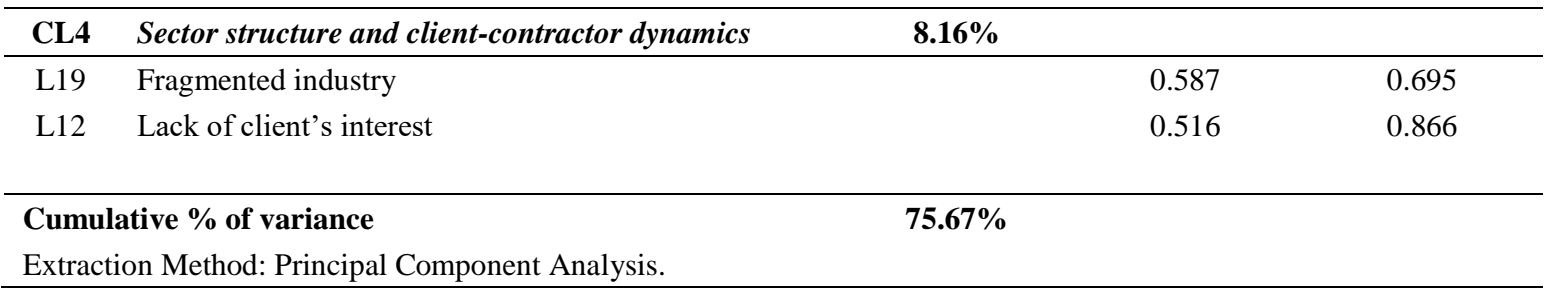

Table 9. Four components extracted that group the factors driving adoption

\begin{tabular}{|c|c|c|c|c|}
\hline Label & Categories and factors & $\begin{array}{c}\text { \% of variance } \\
\left(\% \sigma^{2}\right)\end{array}$ & $\begin{array}{c}\text { Factor loading } \\
(f)\end{array}$ & $\begin{array}{c}\text { Communalities } \\
\text { (extraction) }\end{array}$ \\
\hline CD1 & Improving performance in projects & $22.12 \%$ & & \\
\hline D18 & $\mathrm{AR}$ and VR will reduce overall spending in projects. & & 0.760 & 0.862 \\
\hline D15 & The need to increase labor productivity in the sector. & & 0.730 & 0.831 \\
\hline D21 & AR and VR contributes to better project delivery. & & 0.657 & 0.841 \\
\hline D14 & Difficulties to access labor will drive adoption. & & 0.718 & 0.685 \\
\hline D20 & AR and VR improves collaboration between parties. & & 0.660 & 0.641 \\
\hline D19 & AR and VR improves project understanding. & & 0.611 & 0.834 \\
\hline D16 & Government incentives. & & 0.644 & 0.693 \\
\hline D13 & Decrease in construction budgets will drive adoption. & & 0.545 & 0.752 \\
\hline CD2 & Improving the companies' image & $19.09 \%$ & & \\
\hline D4 & To be part of the trend. & & 0.689 & 0.643 \\
\hline D3 & Obtaining a differentiating advantage in the market. & & 0.672 & 0.827 \\
\hline D2 & Strategic decision from top management. & & 0.544 & 0.828 \\
\hline D7 & Improves the reputation of the organization. & & 0.533 & 0.845 \\
\hline D1 & Client requires the use of $\mathrm{AR} \& \mathrm{VR}$. & & 0.527 & 0.716 \\
\hline CD3 & Improving companies' overall performance & $16.16 \%$ & & \\
\hline D11 & A way to provide new and better services. & & 0.775 & 0.852 \\
\hline D9 & Improves the organization's work culture. & & 0.694 & 0.698 \\
\hline D10 & Enables market expansion. & & 0.664 & 0.818 \\
\hline D5 & Organization's need to be more efficient and productive. & & 0.530 & 0.668 \\
\hline D8 & $\mathrm{AR}$ and VR reduces overall risks. & & 0.516 & 0.798 \\
\hline CD4 & Bolstering research and development & $12.11 \%$ & & \\
\hline D6 & Fostering research curiosity of the employees. & & 0.639 & 0.837 \\
\hline D12 & Increasing $\mathrm{R} \& \mathrm{D}$ investment in the construction sector. & & 0.440 & 0.781 \\
\hline $\mathrm{D} 17 *$ & Adequate marketing of $\mathrm{AR}$ and VR technologies & $7.63 \%$ & 0.581 & 0.747 \\
\hline
\end{tabular}


* Factor D17 was not grouped in any category. This factor in its own has a significant percentage of variance. Extraction Method: Principal Component Analysis.

\section{Limitations for the adoption of $A R$ and VR in the construction industry}

This section provides an explanation of the four extracted categories and the corresponding limiting factors presented in Table 8. Note that PCA only defines groups of correlated factors, but it does not specify the correlations. This section seeks to specify and explain these correlations, considering the construction industry context and dynamics. Note as well that the categories are ordered based on their percentage of variance and not on the importance of their individual factors.

\subsection{Immature technologies}

The main limitation for the adoption of AR and VR in construction is the perception that they are immature technologies that cannot be fully used in practice yet. Battery limitations (The battery of AR headset usually lasts only 30 minutes), narrow fields of view, low tracking accuracy, low resolutions, uncomfortable HMDs are commonly cited factors that disincentivize the use of AR and VR technologies in real-life projects. Construction and engineering applications demand higher levels of accuracy, consistency, and efficacy. For example, the very complex 3D information models commonly used in construction and engineering cannot be handled by current AR devices. In this respect, most of the AR and VR devices have been developed for the entertainment sector; thus, their lack of capabilities for the engineering and construction sectors. Devices that address the specific high-level requirements of professional sectors need to be developed. However, using existing devices for construction and engineering applications is an effective exercise to identify which capabilities need to be improved for the "engineering-level devices" and identify additional ones such as water resistance, ruggedness, etc.

Regarding labor dynamics, the construction industry is not yet a mature field, unlike the entertainment industry. It is very difficult for construction firms to attract AR and VR experts who usually prefer the entertainment and gaming industries. There are not enough graduates with the required skills, and only large firms manage to have teams dedicated to AR and VR development. In addition, the workforce in the construction industry needs to be upskilled, which might represent a huge challenge as, in general the construction industry does not have a trained workforce, and upskilling has not been widely employed (Hampson, Kraatz and Sanchez, 2014).

\subsection{Non-technical issues}

The second category in importance includes factors related to non-technical issues that limit adoption. For example, it is difficult for firms to get access to expert knowledge and advice. Construction firms do not have knowledge of the AR and VR hardware and software market and its dynamics, and it is 
difficult to get advice on plans for procurement and to compare devices. It is difficult for firms to get access to finance and justify the investments required because the cost implications and potential benefits are not clear. The immaturity of the AR and VR market and the lack of expert advice are largely responsible for these issues. Also, AR and VR technologies do not have a good reputation in the construction and engineering field. AR and VR technologies are perceived as technologies for entertainment and with limited potential for complex engineering activities. This notion is rightly based on the factors explained in the previous sub-section. However, this notion does not consider the huge potential benefits that "engineering-grade" AR and VR technologies can bring to the AEC sectors. The idea that AR and VR technologies are only for entertainment limits adoption efforts as not enough time is allowed for experimentation. One approach to counter this notion is to highlight the use of other entertainment tools for engineering applications. For example, game engines have been used to develop simulation environments to train driverless cars (Fayjie et al., 2018) and for structural monitoring (Davila Delgado et al., 2018). Lastly, as with every other digital technology, issues with the aversion to change, job insecurity, data security, and data ownership exist. Such issues are particularly pertinent as the construction industry is known for its poor data management practices (Jonassen, 2010), low digitization (Manyika et al., 2015), and untrained workforce (Castro-Lacouture, 2009).

\subsection{Special requirements for implementation}

The third category includes limiting factors that concern the special requirements needed to adopt AR and VR technologies. VR requires head-mounted displays (HMDs), special controllers, movement tracking sensors and a powerful personal computer with a high-end graphics processing unit. Only specific personal computers and laptops can run VR applications. Mobile VR requires high-end mobile phones and head-mounted adapter. AR requires very expensive HDMs. Mobile AR requires newer versions of mobile phones and tablets. All this equipment can represent a very significant investment, particularly if a wide adoption is planned for which many sets of equipment will be required. More importantly, AR and VR require large spaces or dedicated rooms to set up the equipment. Allocating large empty spaces for AR and VR can represent large costs for small and medium firms.

This category also includes the lack of capabilities to ensure a seamless and robust information exchange. This lack of capabilities is amplified by the poor data management and data exchange practices typical in the construction industry (Vähä et al., 2013; Davila Delgado et al., 2016, 2017), and the impossibility of playing content developed for a specific AR or VR device on another one. This lack of interoperability among AR and VR devices limits adoption greatly as construction firms are forced to choose between different platforms.

Lastly, AR and VR content is experienced by a single individual. This is perceived as a major limitation for adoption even though multiuser capabilities for AR and VR are now in development. For example, 
up to 3 people can wear an AR HMD and experience the same content in the same physical location. In the case of VR, up to approximately 25 people can be in the same virtual room while in different physical locations. Nevertheless, these capabilities have not been fully developed. AR multiuser is limited by the bandwidth of the wireless connection, and more importantly, multiuser experiences in which some people use VR HMDs and others use AR HMDs have not been fully developed yet. The lack of effective multiuser capabilities is the reason why AR and VR are perceived as technologies that can improve communication, but that also increase isolation and inhibit collaboration.

\subsection{Sector structure and client-contractor dynamics}

The last category in importance includes factors related to the structure of the construction industry and the dynamics between clients and contractors. The AEC sectors are highly fragmented, which limits the adoption of emerging digital technologies (Jonassen, 2010; Vähä et al., 2013). Construction projects are delivered by a large and varied group of small companies (Hampson, Kraatz and Sanchez, 2014), and the supply chain is highly fragmented. The successful adoption of digital technologies like AR and VR will require a lower level of fragmentation and alignment of interests within the supply chain. For example, other sectors such as aerospace and automotive also have very complex and varied supply chains; however, the level of fragmentation is considerably lower, and the digitization is considerably higher as well. The other main factor limiting adoption in this category is the lack of client requirements to use AR and VR technologies in projects. Lack of client requirement has been identified as a significant limitation for the uptake of other digital technologies in the AEC sectors as well (Eadie $e t$ al., 2015). The client plays a very important role for adoption, given the high-risk low-profit characteristic of the construction sector (Castro-Lacouture, 2009), in which the adoption of new technologies, productivity, and quality improvements are not a priority.

\section{Drivers for the adoption of $A R$ and VR in the construction industry}

Similar to the previous section, in here an explanation of the four extracted categories and their corresponding limiting factors (Table 9) is presented.

\subsection{Improving performance in projects}

The main driver for the adoption of AR and VR technologies in the AEC sectors is that it can improve the delivery of construction projects. Construction companies recognize that AR and VR can contribute to improve communication and collaboration, improve project understanding, improve productivity, and reduce spending in projects. There is no hard evidence of these benefits in the construction industry, but experiences from other industries are an indication that they can be achieved. For example, Boeing (2018) reported up to a $40 \%$ increase in productivity for assembly tasks using AR and other improvements in productivity for industrial applications have been also reported in literature (e.g. 
Ramírez et al., 2015). Other factors in this category relate to issues that affect project delivery, e.g. the decreasing budgets for construction, the notion of doing more with less, the difficulties in accessing labor, and the lack of government incentives.

\subsection{Improving the companies' image}

The second category includes driving factors related to the potential of AR and VR adoption to improve the image of companies. Nowadays, there is the idea that every successful company is a technology development company. Construction companies are rebranding themselves as smart engineering solutions providers. Construction companies identify the need to adopt digital technologies to improve the reputation of the company. Strong motivators to adopt AR\&VR are the desire to not be left out and to have a differentiating advantage in the market. These motivations are accentuated as large technology companies, e.g. IBM (Murchu, Platt and Webb, 2016) and Oracle (Ali, 2018), are venturing into construction. Their expertise in digital technologies such as Big Data and Artificial Intelligence pose huge potentials to revolutionize the global AEC sectors (Bilal et al., 2016; Davila Delgado et al., 2019); which its global market is expected to reach $\$ 10$ trillion by 2020 (Farnham, 2018). The importance of the construction sector is further emphasized by its share of national GDP, which can represent up to $15 \%$ of GDP in most countries (Oesterreich and Teuteberg, 2016); and the growing venture capital investment on construction technology start-ups, which reached more than $\$ 1$ billion in the first half of 2018 (Jones, Lang and Lasalle, 2018). In this regard, AR and VR adoption can be driven by a strategic decision from top management or by client requirements, as identified by factors D1 and D2 in Table 9. There are indications that suggest that top management decisions on adoption of digital technologies are very effective on driving adoption; as highly-digitized companies closely tie their digital and corporate strategies and adapt significantly their corporate strategies to the adoption of new digital technologies (Bughin, LaBerge and Mellbye, 2017).

\subsection{Improving companies' overall performance}

This category includes the factors representing the desire of companies to strive for the success of the company as a whole. The adoption of AR and VR technologies is regarded as a way to provide new and better services and to expand to other the markets. For example, companies from sectors ranging from commerce consultancies to retail and beauty products, are investing significantly in AR and VR technologies. It is also seen as a way to improve the organization's work culture and to increase overall productivity. The adoption of any emerging digital technology can contribute to improving work culture in organizations (Buchanan, Kelley and Hatch, 2016). The adoption of AR and VR is also considered as a way to reduce risks, which is very important for the construction industry, as is regarded as a highrisk sector (Castro-Lacouture, 2009). 


\subsection{Bolstering research and development}

Bolstering Research and Development (R\&D) is the last category of factors that drive adoption, which includes factors D6 (Fostering research curiosity of the employees) and D12 (Increasing $R \& D$ investment in the construction sector). The construction industry has not invested sufficiently in R\&D (Hampson and Newton, 2009; Bock, 2015), and the scope of the research carried out is too narrow to foster step-change innovations (Forbes and Ahmed, 2011). The lack of sufficient investment in R\&D and its narrow scope contribute to a weak innovation culture in the construction industry (Manley et al., 2008). This is exemplified by the remarkably low adoption of digital technologies in the construction industry, as its digitization index is one of the lowest out of 22 different industries surveyed (Manyika et al., 2015). There are strong indications from examples in other sectors, such as automotive and aerospace (Gandhi, Khanna and Ramaswamy, 2016), that increasing the R\&D investment in the AEC sectors will drive adoption of digital technologies including AR and VR. Globally, the investment in $R \& D$ in construction has doubled in the last decade (Blanco et al., 2018). The UK construction industry has increased its R\&D investments 18-fold in last 11 years up to 2017 (Prescott, 2018); moving construction from the bottom of the R\&D sector list to an intermediate position. Still, the UK aerospace industry R\&D investment is $\sim 4$ times larger and the automotive is $\sim 10$ times bigger (Prescott, 2018). The levels of R\&D investment in the construction industry must continue and accelerate to catch up with the other leading sectors. Notably, the results of this study also indicate that resources - time in particular - must be allocated to foster and enable workers to experiment with emerging technologies. During the FGDs, anecdotical evidence was provided, which indicated that the adoption of AR and VR yielded better results when it was driven by a group of enthusiastic workers with enough freedom and time than by a top management decision.

Note that factor D17 is not grouped in any of the categories above because is not strongly correlated to them. This factor is the fifth in importance representing a 7.63\% percentage of the variance. Factor D17 identifies that adequate branding and marketing strategies to present AR and VR solutions as capable of supporting construction and engineering tasks would benefit uptake.

\section{Discussion}

The main limitation for adoption is that AR and VR technologies are regarded as expensive and immature technologies. To tackle these issues, $R \& D$ efforts should be focused on developing technologies for the specific requirements of the AEC sectors. Given the large investments required to implement AR and VR in terms of equipment, space, time, and upskilling; only by developing AR and VR hardware and software specific for the AEC sectors the investments can be justified. In this respect, further studies are needed to identify what capabilities are required for the AEC sectors and are missing in current AR and VR hardware and software. R\&D should address non-technical issues as well. Better AR and VR devices will not be enough. Evidence of accrued value from real-life projects is required as 
well. Detailed cost-benefit studies and real-life demonstrators have the potential to provide evidence and improve the reputation of the technologies. Future research should consider a more granular study regarding the limitations of AR and VR technologies for specific construction tasks and identify in which phases of the built asset life cycle the implementation is easier and in which phases represent the largest benefits for adoption.

Improvements in project delivery and providing new and better services are the main drivers for adoption. R\&D efforts should focus on boosting and showcasing these factors. For example, (i) developing workflows that integrate $\mathrm{AR}$ and VR into current standard practices, (ii) developing new business models that leverage AR and VR capabilities, and, most importantly, (iii) defining actions to develop a digitally empowered workforce. A study by Gandhi et al., (2016), identified providing digital tools in the hands of their employees is the a key factor to ramp up productivity. In this respect, promoting the use of AR and VR can kick-start a virtuous cycle, in which adoption of AR and VR can improve productivity and the adoption of other emerging digital technologies as well. A more digitized construction industry will potentially help addressing the massive labor shortage in the construction industry as well. For example, in the US there were $\sim 430,000$ vacant construction jobs as of April 2019 (US-Labor-Bureau, 2019). Nowadays, workers avoid construction jobs, perceiving them as dangerous, difficult, and dirty, as they prefer take on jobs in retail or transportation (Cilia, 2019). A more digitized construction industry will make the industry more appealing and help attract young talent.

Lastly, stakeholders should explore alternative and innovative use-cases of AR and VR, which will help to justify the investments required to adopt AR and VR. A detailed agenda on future research, new capabilities and innovative use-cases has been presented in a recent report by the authors (Davila Delgado et al., 2019a). Some of the most notable are: (i) AR and VR teleoperation and plant control (Lipton et al., 2018), (ii) diminished reality (Mori, Ikeda and Saito, 2017), and (iii) AR and VR archival (Hahn et al., 2019).

Regarding implications for practice, this study provides stakeholders with a manageable number of categories of limiting and driving factors. These categories are explained, and insights are provided considering the specific context and dynamics of the construction industry. Stakeholders can use these insights to devise actions to mitigate the limiting factors and to boost the driving factors. For example, stakeholders can use the information provided in this study to define specific strategies to facilitate the adoption of AR and VR within organizations and to educate clients as well. In this regard, Section 8.1 presents a set of short-term and medium-term actions that can help stakeholders to device an action plan to facilitate AR and VR adoption. 


\subsection{Roadmap for improving adoption}

Based on the limiting factors and informed by the driving factors presented in Tables 8 and 9 , a roadmap to improve adoption of AR and VR technologies has been developed. This roadmap sets out a series of short-term and medium-term actions that AEC companies can carry out to increase the adoption of AR and VR technologies within the built environment. The timescales in this table are derived by factoring in the importance of the limitation and the feasibility of its resolution. Short-term refers to actions that can be carried out within a year, and medium-term refers to actions to be carried out within 3 years.

The short-term actions are: (i) Increased training opportunities for AEC professionals in AR and VR. Many AEC professionals lack skills in these technologies, and increased training opportunities are required to fill this gap. This can be achieved through increased continuing professional development opportunities and integration of AR and VR skills within university education. Note that digitally engaged workforces are a crucial factor for success of leading companies, which can have employees that are more than 10 times more engaged with digital technologies (Gandhi, Khanna and Ramaswamy, 2016). (ii) Increased access to expert knowledge. Many AEC organizations lack expert knowledge to properly leverage AR and VR technologies. To overcome this, a directory of consultants and other organizations able to support organizations through the adoption of these technologies should be established. Additionally, development of in-house expertise should be fostered by attracting talent from other sectors and upskilling of the current workforce. (iii) Correction of industry perceptions and better branding of AR and VR technologies. Industry perceptions of AR and VR technologies are often that they are primarily for "gaming" purposes. To widen the adoption of these technologies this perception should be dispelled through an awareness driving initiative and an improved branding of products to signify their professional usage. (iv) Increasing client awareness and decreasing aversion to the possibilities of $A R$ and $V R$. Construction clients are generally unaware of the benefits of AR and VR technologies and thus reluctant to include this within project costs. Awareness driving activities are required to overcome this.

The medium-term actions are: (i) Implementation of systematic and semi-automated workflows to create $A R$ and VR content. AEC companies that have already tested AR and VR should invest in developing automated workflows that facilitate the use of BIM models and project data to generate AR and VR content. Developing systematic and semi-automated workflows facilitates greatly content creation and will increase adoption. For example, the work of Du et al. (2018b) can potentially be very useful to enable real-time synchronization of BIM data with VR applications. (ii) Implementation of data exchange standards and open-source conversion tools. Current AR and VR technologies are not compatible with AEC standard data exchange formats (e.g. Industry Foundation Classes), making the integration of standard AEC software packages and AR and VR software tools difficult. Thus, AEC companies should engage and aid standardization bodies to include support for AR and VR formats. 
AEC companies should also collaborate on developing open-source conversion tools between AEC file formats and AR and VR formats. In this case, the work of Dris et al. (2019) is very relevant as ontologies that enable bi-directional links between the BIM models and VR applications are essential to ensure robust data exchanges. (iii) Increased support for data security and ownership matters. AR and VR toolchains do not generally address information security and privacy issues out of the box. These are key concerns for many use cases, and AR and VR software tools need to be expanded to provide support for security and privacy out of the box. AEC companies must ask software providers to better support these cases.

\subsection{Comparison with similar studies}

Recently there has been a big increase in research reported in literature regarding AR and VR. However, there are no relevant studies that investigate the factors limiting and driving the adoption of both AR and VR in the construction industry. Behzadan et al. (2015) presented a literature review that focuses only on AR for civil infrastructure. The study focuses only on the technical aspects of AR implementation. The authors identify the two main technical problems that AR solutions face for implementation, i.e.: (1) the registration problem, which relates to the difficulties to solve the spatial alignment of real and virtual entities. (2) The occlusion problem, which relates to the visual illusions required so that the virtual and real-world coexist in a credible manner. The main use cases identified by the authors are: support with damage identification, localization of buried elements and support for collaborative design. Li et al. (2018) considered AR and VR together, however, the literature review focuses only on construction safety, for which articles published between 2000 and 2017 were analyzed. The authors conclude that the top applications reported in the literature include hazards identification, safety education and training, and safety inspection and instruction. Guo et al. (2017) also presented a review on visualization methods for construction safety management, which addressed AR and VR only in a limited manner. Palmarini et al. (2018) presented a technical review of AR research reported in literature for industrial maintenance tasks in various fields such as aviation, nuclear, consumer technology and plant and mechanical maintenance. The authors conclude that AR is still not sufficiently mature and reliable to comply with industrial requirements. These studies are the most closely related to the investigation presented here. However, the studies above are focused only on a limited set of applications and do not focus on the factors that limit or drive adoption. More importantly, all the studies above arrive at conclusions only based on literature analyses, which limits the amount of information that can be collected and analyzed. The study presented here uses a more robust research method that enables to capture more information with practical relevance about the actual factors limiting and driving adoption. The information was captured directly from practitioners across various types and sizes of AEC companies, which reflects the actual structure of the construction sector in practice. Therefore, the findings presented in this study can capture the whole complex dynamic of AR and VR 
adoption more effectively. For example, this study describes the non-technical issues that limit adoption, it addresses how the structure and dynamics of the construction sector may affect adoption, and, it defines the factors that AEC companies can leverage to drive adoption; which none of the other studies addressed.

Reviews and studies on AR and VR in other fields have been carried out recently as well. Here, examples from Tourism, Healthcare, and Education are presented, which arrived at similar conclusions as this study. This is an indication of the relevance and the potential generalization of the findings presented in this study. Yung and Khoo-Lattimore (2017) presented a literature review on AR and VR research in Tourism. The authors identified the following use cases (i) marketing, (ii) tourism education, (iii) experience enhancement, (iv) improved communication among individuals, and (v) food safety training. The authors note that there are prevalent issues such as a lack of awareness of the technology, poor usability, large time commitment for implementation, and the unwillingness to accept a virtual substitute. Glegg and Levac (2018) presented a scoping review to identify barriers and facilitators to support the implementation of VR in medical rehabilitation. The identified barriers and facilitators were grouped in three categories (1) technology development, which is the level that the technology can meet the user's objectives or needs; (2) competency development, which is the level of technical skills required to use the technology clinically effectively and safely; and (3) clinical implementation, which is the set of technical, time, training, and spatial requirements necessary to implement the technology. The authors note that there is the need to study the actual effectiveness of the technology; and for targeted development of implementation research, which should help with the development testing and implementation processes. Akçayır and Akçayır (2017) presented a literature review that studied, among other topics, the challenges for AR adoption in education. The authors note that the most common problems are (i) the usability difficulties for students to use AR applications, (ii) the additional time requirements, (iii), and the lack of robustness and sensitivity to trigger AR interactions. Ibáñez and Delgado-Kloos (2018) also presented a review, but in this case, it focused on the effects of AR on students. The authors note that there are indications that AR could promote distraction and that it increases cognitive loads. Similar studies should be carried out in the construction sector as well to identify potential negative effects that AR and VR can have on the workers' performance.

\subsection{Limitations of this study}

The main limitation is that the UK was the focus of the study and only academics and professionals based in the UK were engaged. Therefore, the results presented here are not entirely generalizable to other regions (e.g. North America, South America, Europe, Australia, and Asia, etc.) due to the different structures, dynamics, markets, stakeholders, companies, clients, labor, etc. of construction industries in different regions. In this sense, that main limiting and driving factors and the categories presented here might be different in different parts of the world. Hence, other studies that investigate the limiting and 
driving factors in other parts of the world are required. These studies will help to (i) identify regional differences in the limiting and driving factors, (ii) identify differences in their importance and categorization, and (iii) validate the results presented here.

However, the findings and insights presented in this paper could be relevant for AEC practitioners and academics across the developed world, because (i) studies have found existing similarities among the construction industries of developed economies. For example, Barbosa et al. (2017) found that the construction industry's labor productivity and labor productivity growth are very similar in most European countries, the US, Australia, and Israel. (ii) Many of the experts that participated in this study work for transnational companies and they are either based-on or have experience working in developed economies, including the US, Canada, and the EU. Therefore, the experience of the international participants enriched and broaden the findings of the study presented here.

More importantly, as detailed in section 8.1, other studies on AR and VR adoption carried out in other fields (Tourism, Healthcare, and Education) arrived at similar conclusions as this study. This is an indication of the relevance and the potential generalization of the findings here. For example, Yung and Khoo-Lattimore (2017), found that the main limiting factors for adoption are lack of awareness of the technology, poor usability, large time commitment for implementation, and the unwillingness to accept a virtual substitute; which all have been identified in this study as well.

Overall, this study can be useful for practitioners and academics outside the UK by providing (i) a good indication of what type of factors could be important for adoption, (ii) an example of AR and VR adoption in the construction industry of a developed economy, (iii) and an example of a methodology that can be used in other regions to identify which limiting and driving factors are at play locally.

\section{Conclusions}

This paper presented a mixed research study into the factors that limit and drive the adoption of AR and VR in the construction industry. The two main objectives of this paper were (i) to identify, categorize, and rank the relevant factors that limit and drive adoption of AR and VR and (ii) to provide a clear and understandable explanation of these factors to use as the basis to develop mitigating actions. Exploratory workshops and focus group discussions were carried out, in which 54 experts participated from 36 UK organizations from industry and academia. Twenty-one limiting factors and twenty-one driving factors were identified. The importance of the factors was ranked using a quantitative tool and statistical methods. The most important limiting factor is the high cost for equipment and training, and the most important driving factor is that this technology enables new and better services to be provided. PCA was carried out to identify the correlations between the two sets of factors and define a smaller number of manageable factors. Four categories of limiting factors were defined, i.e.: (1) Immature technologies, (2) Non-technical issues, (3) Specific requirements for implementation, and (4) Sector 
structure and client-contractor dynamics. Four categories of driving factors were also defined, i.e.: (i) Improving performance in projects, (ii) Improving the companies' image, (iii) Improving companies' overall performance (iv) Bolstering research and development. The main limitation of adoption is that $\mathrm{AR}$ and VR technologies are regarded as expensive and immature technologies. Improvements in project delivery and providing new and better services are the main drivers for adoption. The complex context and dynamics of the construction sector limit the adoption of AR and VR. This study presented a systematic study that contributes to identifying the essential and underlying factors and provides the insights required to devise effective actions to drive adoption. Finally, a roadmap is proposed to implement key short-term and medium-term actions to help overcome these factors. The main contribution to knowledge of this study is that it grouped and characterized a myriad of limiting and driving factors into easily understandable categories; so that, the limiting factors can be effectively mitigated, and the driving factors potentiated.

\section{Data Availability Statement}

Data generated or analyzed during the study are available from the corresponding author by request.

\section{Acknowledgements}

The authors would like to gratefully acknowledge the Cambridge Centre for Digital Built Britain (CDBB) for funding this research, under the Vision Network project via Innovate UK and the Department for Business, Energy and Industrial Strategy. The contribution of the Vision Network core members Stephané Côte, Andrew Gamblen, Amer Hijazi, Andrew Jordaan, Mac Muzviwe, Hasan Omar, Hadeel Sadoon, Mohammad Samie and Zakwan Skaf is also acknowledged.

\section{References}

Ahn, S., Han, S. and Al-Hussein, M. (2019) '2D Drawing Visualization Framework for Applying Projection-Based Augmented Reality in a Panelized Construction Manufacturing Facility: Proof of Concept', Journal of Computing in Civil Engineering, 33(5), p. 04019032. doi: 10.1061/(ASCE)CP.1943-5487.0000843.

Akçayır, M. and Akçayır, G. (2017) 'Advantages and challenges associated with augmented reality for education: A systematic review of the literature', Educational Research Review. Elsevier, 20, pp. 1-11. doi: 10.1016/J.EDUREV.2016.11.002.

Albert, A. et al. (2014) 'Enhancing Construction Hazard Recognition with High-Fidelity Augmented Virtuality', Journal of Construction Engineering and Management, 140(7), p. 04014024. doi: 10.1061/(ASCE)CO.19437862.0000860 .

Ali, B. (2018) The Silicon Valley giant that wants to reinvent construction, Construction News. Available at: https://www.constructionnews.co.uk/best-practice/technology/the-silicon-valley-giant-that-wants-to-reinventconstruction/10034264.article (Accessed: 8 February 2019).

Annetta, L. et al. (2009) 'Bridging Realty to Virtual Reality: Investigating gender effect and student engagement on learning through video game play in an elementary school classroom', International Journal of Science Education. Routledge , 31(8), pp. 1091-1113. doi: 10.1080/09500690801968656.

Aromaa, S. and Väänänen, K. (2016) 'Suitability of virtual prototypes to support human factors/ergonomics evaluation during the design', Applied Ergonomics. Elsevier, 56, pp. 11-18. doi: 10.1016/J.APERGO.2016.02.015.

Baek, F., Ha, I. and Kim, H. (2019) 'Augmented reality system for facility management using image-based indoor localization', Automation in Construction. Elsevier, 99, pp. 18-26. doi: 10.1016/J.AUTCON.2018.11.034. 
Barbosa, F. et al. (2017) Reinventing construction through a productivity revolution. Available at: https://www.mckinsey.com/industries/capital-projects-and-infrastructure/our-insights/reinventing-constructionthrough-a-productivity-revolution (Accessed: 22 June 2019).

Behzadan, A. H., Dong, S. and Kamat, V. R. (2015) 'Augmented reality visualization: A review of civil infrastructure system applications’, Advanced Engineering Informatics. Elsevier, 29(2), pp. 252-267. doi: 10.1016/J.AEI.2015.03.005.

Behzadi, A. (2016) 'Using Augmented and Virtual Reality Technology in the Construction Industry', American Journal of Engineering Research, 5(12), pp. 350-353. Available at: https://pdfs.semanticscholar.org/2fc8/2c3dfd09f7439fa75a6015c5a8eea1ff19fc.pdf (Accessed: 30 January 2019).

Benford, S. et al. (1998) 'Understanding and constructing shared spaces with mixed-reality boundaries', ACM Transactions on Computer-Human Interaction. ACM, 5(3), pp. 185-223. doi: 10.1145/292834.292836.

Berg, L. P. and Vance, J. M. (2016) 'An Industry Case Study: Investigating Early Design Decision Making in Virtual Reality', Journal of Computing and Information Science in Engineering. American Society of Mechanical Engineers, 17(1), p. 011001. doi: 10.1115/1.4034267.

Bilal, M. et al. (2016) 'Big Data in the construction industry: A review of present status, opportunities, and future trends', Advanced Engineering Informatics. Elsevier Ltd, 30(3), pp. 500-521. doi: 10.1016/j.aei.2016.07.001.

Billinghurst, M. and Kato, H. (2002) 'Collaborative augmented reality', Communications of the ACM. ACM, 45(7), pp. 64-70. doi: 10.1145/514236.514265.

Blanco, J. L. et al. (2018) Seizing opportunity in today's construction technology ecosystem. Available at: $\mathrm{https}$ ///www.mckinsey.com/industries/capital-projects-and-infrastructure/our-insights/seizing-opportunity-in-todaysconstruction-technology-ecosystem.

Bock, T. (2015) 'The future of construction automation: Technological disruption and the upcoming ubiquity of robotics', Automation in Construction. Elsevier, 59, pp. 113-121. doi: 10.1016/J.AUTCON.2015.07.022.

Boeing (2018) Boeing Tests Augmented Reality in the Factory, Boeing Innovation and Technology. Available at: https://www.boeing.com/features/2018/01/augmented-reality-01-18.page (Accessed: 7 February 2019).

Boton, C. (2018) 'Supporting constructability analysis meetings with Immersive Virtual Reality-based collaborative BIM 4D simulation', Automation in Construction. Elsevier, 96, pp. 1-15. doi: 10.1016/J.AUTCON.2018.08.020.

Boud, A. C. et al. (1999) 'Virtual reality and augmented reality as a training tool for assembly tasks', in 1999 IEEE International Conference on Information Visualization (Cat. No. PR00210). IEEE Comput. Soc, pp. 32-36. doi: 10.1109/IV.1999.781532.

Bray, B. and Zeller, M. (2018) What is mixed reality? Microsoft. Available at: https://docs.microsoft.com/enus/windows/mixed-reality/mixed-reality.

Buchanan, J., Kelley, B. and Hatch, A. (2016) Digital workplace and culture: How digital technologies are changing the workforce and how enterprises can adapt and evolve.

Bughin, J., LaBerge, L. and Mellbye, A. (2017) The case for digital reinvention. Available at: https://www.mckinsey.com/business-functions/mckinsey-digital/our-insights/the-case-for-digital-reinvention.

Castro-Lacouture, D. (2009) 'Construction Automation', in Springer Handbook of Automation. Berlin, Heidelberg: Springer Berlin Heidelberg, pp. 1063-1078. doi: 10.1007/978-3-540-78831-7_61.

Chalhoub, J. and Ayer, S. K. (2018) 'Using Mixed Reality for electrical construction design communication', Automation in Construction. Elsevier, 86, pp. 1-10. doi: 10.1016/J.AUTCON.2017.10.028.

Chu, M., Matthews, J. and Love, P. E. D. (2018) 'Integrating mobile Building Information Modelling and Augmented Reality systems: An experimental study', Automation in Construction. Elsevier, 85, pp. 305-316. doi: 10.1016/J.AUTCON.2017.10.032.

Cilia, J. (2019) The Construction Labor Shortage: Will Developers Deploy Robotics?, Forbes. Available at: https://www.forbes.com/sites/columbiabusinessschool/2019/07/31/the-construction-labor-shortage-will-developersdeploy-robotics/\#1d0df4e71988.

Creswell, J. (2017) Research design: Qualitative, quantitative, and mixed methods approaches. Sage Publications. Available at: https://scholar.google.com/scholar_lookup?title=Research Design\%3A Qualitative\%2C Quantitative\%2C and Mixed Methods Approaches\&author=J.W. Creswell\&publication_year=2014 (Accessed: 30 October 2018).

Davila Delgado, J. M. et al. (2017) 'Management of structural monitoring data of bridges using BIM', Proceedings of the Institution of Civil Engineers - Bridge Engineering. Thomas Telford Ltd, 170(3), pp. 204-218. doi: 10.1680/jbren.16.00013.

Davila Delgado, J. M. et al. (2018) 'Structural performance monitoring using a dynamic data-driven BIM environment', Journal of Computing in Civil Engineering, 32(3). 
Davila Delgado, Juan Manuel et al. (2019) 'Big Data analytics system for costing power transmission projects', Journal of Construction Engineering and Management, in press.

Davila Delgado, J.M. et al. (2019a) Vision Network Augmented Reality and Virtual Reality for Digital Built Britain. doi: https://doi.org/10.17863/CAM.40454.

Davila Delgado, J. M., Brilakis, I. and Middleton, C. R. (2016) 'Modelling, management, and visualisation of structural performance monitoring data on BIM', in Transforming the Future of Infrastructure through Smarter Information, Proceedings of the International Conference on Smart Infrastructure and Construction, pp. 543-549. Available at:

https://www.researchgate.net/profile/Ioannis_Brilakis/publication/305303327_Modelling_management_and_visualisat ion_of_structural_performance_monitoring_data_on_BIM/links/578754e708aea50b6b18f 48d.pdf.

DeFanti, T. A. et al. (2009) 'The StarCAVE, a third-generation CAVE and virtual reality OptIPortal', Future Generation Computer Systems. North-Holland, 25(2), pp. 169-178. doi: 10.1016/J.FUTURE.2008.07.015.

Deshpande, A. and Kim, I. (2018) 'The effects of augmented reality on improving spatial problem solving for object assembly', Advanced Engineering Informatics. Elsevier, 38, pp. 760-775. doi: 10.1016/J.AEI.2018.10.004.

Dong, S. et al. (2013) 'Collaborative visualization of engineering processes using tabletop augmented reality', Advances in Engineering Software. Elsevier, 55, pp. 45-55. doi: 10.1016/J.ADVENGSOFT.2012.09.001.

Dris, A.-S. et al. (2019) 'OpenBIM Based IVE Ontology: An Ontological Approach to Improve Interoperability for Virtual Reality Applications', in Advances in Informatics and Computing in Civil and Construction Engineering. Cham: Springer International Publishing, pp. 129-136. doi: 10.1007/978-3-030-00220-6_16.

Du, J., Shi, Y., et al. (2018) 'CoVR: Cloud-Based Multiuser Virtual Reality Headset System for Project Communication of Remote Users', Journal of Construction Engineering and Management, 144(2), p. 04017109. doi: 10.1061/(ASCE)CO.1943-7862.0001426.

Du, J., Zou, Z., et al. (2018) 'Zero latency: Real-time synchronization of BIM data in virtual reality for collaborative decision-making', Automation in Construction. Elsevier, 85, pp. 51-64. doi: 10.1016/J.AUTCON.2017.10.009.

Dunston, P. S. et al. (2011) 'An Immersive Virtual Reality Mock-Up for Design Review of Hospital Patient Rooms', in Collaborative Design in Virtual Environments. Dordrecht: Springer Netherlands, pp. 167-176. doi: 10.1007/97894-007-0605-7_15.

Eadie, R. et al. (2015) 'A survey of current status of and perceived changes required for BIM adoption in the UK', Built Environment Project and Asset Management. Emerald Group Publishing Limited , 5(1), pp. 4-21. doi: 10.1108/BEPAM-07-2013-0023.

Eiris Pereira, R. et al. (2019) 'Development and Usability Testing of a Panoramic Augmented Reality Environment for Fall Hazard Safety Training', in Advances in Informatics and Computing in Civil and Construction Engineering. Cham: Springer International Publishing, pp. 271-279. doi: 10.1007/978-3-030-00220-6_33.

Ergan, S. et al. (2019) 'Quantifying Human Experience in Architectural Spaces with Integrated Virtual Reality and Body Sensor Networks', Journal of Computing in Civil Engineering, 33(2), p. 04018062. doi:

10.1061/(ASCE)CP.1943-5487.0000812.

Farnham, D. (2018) Global Construction Industry Expected to Reach $\$ 10$ trillion by 2020, Case Study Research: Design \& Methods: Applied Social Research Methods Series. Available at: http://scalar.usc.edu/works/farnhamresearch/global-construction-industry-expected-to-reach-10-trillion-by-2020 (Accessed: 8 February 2019).

Fayjie, A. R. et al. (2018) 'Driverless Car: Autonomous Driving Using Deep Reinforcement Learning in Urban Environment', in 2018 15th International Conference on Ubiquitous Robots (UR). IEEE, pp. 896-901. doi: 10.1109/URAI.2018.8441797.

Fazel, A. and Izadi, A. (2018) 'An interactive augmented reality tool for constructing free-form modular surfaces', Automation in Construction. Elsevier, 85, pp. 135-145. doi: 10.1016/J.AUTCON.2017.10.015.

Fetters, M. D., Curry, L. A. and Creswell, J. W. (2013) 'Achieving Integration in Mixed Methods Designs-Principles and Practices', Health Services Research. Wiley/Blackwell (10.1111), 48(6pt2), pp. 2134-2156. doi: 10.1111/14756773.12117.

Fogarty, J., McCormick, J. and El-Tawil, S. (2018) 'Improving Student Understanding of Complex Spatial Arrangements with Virtual Reality', Journal of Professional Issues in Engineering Education and Practice, 144(2), p. 04017013. doi: 10.1061/(ASCE)EI.1943-5541.0000349.

Forbes, L. H. and Ahmed, S. M. (2011) Modern construction : lean project delivery and integrated practices. CRC Press.

Friedrich, W., Jahn, D. and Schmidt, L. (2002) 'ARVIKA: augmented reality for development, production and service', in ISMAR 2002. New York, New York, USA: ACM Press, pp. 151-152. doi: 10.1145/354666.354688.

Gandhi, P., Khanna, S. and Ramaswamy, S. (2016) ‘Which Industries Are the Most Digital (and Why)?', Harvard 
Business Review, April. Available at: https://hbr.org/2016/04/a-chart-that-shows-which-industries-are-the-mostdigital-and-why.

Glegg, S. M. N. and Levac, D. E. (2018) 'Barriers, Facilitators and Interventions to Support Virtual Reality Implementation in Rehabilitation: A Scoping Review', $P M \& R$. No longer published by Elsevier, 10(11), pp. 12371251.e1. doi: 10.1016/J.PMRJ.2018.07.004.

Golparvar-Fard, M., Peña-Mora, F. and Savarese, S. (2009) 'D4AR a 4-dimensional augmented reality model for automating construction progress monitoring data collection, processing and communication', Journal of information technology in construction, 14(13), pp. 129-153. Available at: https://pdfs.semanticscholar.org/338e/04a889d11a6c6d46827dada3e67dda1833ce.pdf (Accessed: 30 January 2019).

GSA, . (2017) Virtual and Augmented Reality Initiative, GSA IT Initiatives. Available at:

https://www.gsa.gov/technology/government-it-initiatives/emerging-citizen-technology/virtual-and-augmented-reality (Accessed: 21 June 2019).

Guo, H., Yu, Y. and Skitmore, M. (2017) 'Visualization technology-based construction safety management: A review', Automation in Construction. Elsevier, 73, pp. 135-144. doi: 10.1016/J.AUTCON.2016.10.004.

Hahn, J. et al. (2019) 'Institutionalizing and Sustaining Virtual Reality Experiences', in 2019 ACM/IEEE Joint Conference on Digital Libraries (JCDL). IEEE, pp. 325-326. doi: 10.1109/JCDL.2019.00053.

Hampson, K. D., Kraatz, J. A. and Sanchez, A. (2014) 'The global construction industry and R\&D', in $R \& D$ Investment in the global construction industry, pp. 1-16.

Hampson, K. and Newton, P. (2009) 'Transforming the built environment through construction innovation', in Technology, design and process innovation in the built environment. Spoon Press, pp. 29-54. Available at: https://www.taylorfrancis.com/books/e/9781134041831/chapters/10.4324\%2F9780203928325-9 (Accessed: 2 October 2018).

Hassan, H., Taib, N. and Rahman, Z. A. (2018) 'Virtual Design and Construction: a new communication in construction industry', in Proceedings of the 2nd International Conference on Digital Signal Processing - ICDSP 2018. New York, New York, USA: ACM Press, pp. 110-113. doi: 10.1145/3193025.3193062.

Heather Bellini (2016) The Real Deal with Virtual and Augmented Reality, Golman Sacks Technology. Available at: https://www.goldmansachs.com/insights/pages/virtual-and-augmented-reality.html (Accessed: 7 February 2019).

Hofmann, H., Wickham, H. and Kafadar, K. (2017) 'Letter-Value Plots: Boxplots for Large Data', Journal of Computational and Graphical Statistics. Taylor \& Francis, 26(3), pp. 469-477. doi: 10.1080/10618600.2017.1305277.

Ibáñez, M.-B. and Delgado-Kloos, C. (2018) ‘Augmented reality for STEM learning: A systematic review', Computers \& Education. Pergamon, 123, pp. 109-123. doi: 10.1016/J.COMPEDU.2018.05.002.

Jonassen, J. O. (2010) Report on integrated practice. Changing business models in BIM-driven integrated practice.

Jones, J., Lang, J. and Lasalle, J. (2018) The state of construction technology. Available at: http://jll.postclickmarketing.com/construction-technology\#Section-2A (Accessed: 8 February 2019).

Kaiser, R. and Scatsky, D. (2017) For more companies, new ways of seeing, Deloitte Insights. Available at: https://www2.deloitte.com/insights/us/en/focus/signals-for-strategists/augmented-and-virtual-reality-enterpriseapplications.html (Accessed: 7 February 2019).

Kvale, S. (1994) Interviews: An introduction to qualitative research interviewing. Sage Publications. Available at: https://psycnet.apa.org/record/1996-97829-000 (Accessed: 1 March 2019).

$\mathrm{Li}$, X. et al. (2018) 'A critical review of virtual and augmented reality (VR/AR) applications in construction safety', Automation in Construction. Elsevier, 86, pp. 150-162. doi: 10.1016/J.AUTCON.2017.11.003.

Lin, J.-R. et al. (2019) 'Visualization of indoor thermal environment on mobile devices based on augmented reality and computational fluid dynamics', Automation in Construction. Elsevier, 103, pp. 26-40. doi: 10.1016/J.AUTCON.2019.02.007.

Lin, Y.-C. et al. (2018) 'Integrated BIM, game engine and VR technologies for healthcare design: A case study in cancer hospital', Advanced Engineering Informatics. Elsevier, 36, pp. 130-145. doi: 10.1016/J.AEI.2018.03.005.

Lipton, J. I., Fay, A. J. and Rus, D. (2018) 'Baxter's Homunculus: Virtual Reality Spaces for Teleoperation in Manufacturing', IEEE Robotics and Automation Letters, 3(1), pp. 179-186. doi: 10.1109/LRA.2017.2737046.

Lovreglio, R. et al. (2018) 'Prototyping virtual reality serious games for building earthquake preparedness: The Auckland City Hospital case study', Advanced Engineering Informatics. Elsevier, 38, pp. 670-682. doi: 10.1016/J.AEI.2018.08.018.

Manley, K. et al. (2008) 'The potential contribution of small firms to innovation in the built environment'. Available at: http://eprints.qut.edu.au/17182 (Accessed: 2 October 2018).

Manyika, J. et al. (2015) Digital America: A tale of haves and have-mores. 
Milgram, P. (1994) ‘A Taxonomy of Mixed Reality Visual Displays', IEICE Transactions on Information Systems, E77-D(12). Available at: http://vered.rose.utoronto.ca/people/paul_dir/IEICE94/ieice.html (Accessed: 12 September 2018).

Mori, S., Ikeda, S. and Saito, H. (2017) 'A survey of diminished reality: Techniques for visually concealing, eliminating, and seeing through real objects', IPSJ Transactions on Computer Vision and Applications. SpringerOpen, 9(1), p. 17. doi: 10.1186/s41074-017-0028-1.

Morrison, D. F. (2005) 'Multivariate Analysis of Variance', in Encyclopedia of Biostatistics. Chichester, UK: John Wiley \& Sons, Ltd. doi: 10.1002/0470011815.b2a13045.

Motamedi, A. et al. (2017) 'Signage visibility analysis and optimization system using BIM-enabled virtual reality (VR) environments', Advanced Engineering Informatics. Elsevier, 32, pp. 248-262. doi: 10.1016/J.AEI.2017.03.005.

MTC (2017) VR, AR and MR discovery project. Available at: http://www.the-mtc.org/our-projects/i3p-programme.

Mujber, T. S., Szecsi, T. and Hashmi, M. S. J. (2004) 'Virtual reality applications in manufacturing process simulation', Journal of Materials Processing Technology. Elsevier, 155-156, pp. 1834-1838. doi: 10.1016/J.JMATPROTEC.2004.04.401.

Murchu, E., Platt, D. and Webb, G. (2016) The performance advantages of digitizing the built environment. Available at: https://www-01.ibm.com/common/ssi/cgi-bin/ssialias?htmlfid=PQW12354USEN (Accessed: 8 February 2019).

National Infrastructure Commission (2017) Data for the Public Good. Available at: https://www.nic.org.uk/wpcontent/uploads/Data-for-the-Public-Good-NIC-Report.pdf (Accessed: 12 September 2018).

Nee, A. Y. C. et al. (2012) 'Augmented reality applications in design and manufacturing', CIRP Annals. Elsevier, 61(2), pp. 657-679. doi: 10.1016/J.CIRP.2012.05.010.

Neges, M. et al. (2017) 'Combining visual natural markers and IMU for improved AR based indoor navigation', Advanced Engineering Informatics. Elsevier, 31, pp. 18-31. doi: 10.1016/J.AEI.2015.10.005.

Neges, M. and Koch, C. (2016) 'Augmented reality supported work instructions for onsite facility maintenance'. Available at: http://eprints.nottingham.ac.uk/34761/ (Accessed: 30 January 2019).

Nunnally, J. C. and Bernstein, I. H. (1994) Psychometric theory. McGraw-Hill. Available at: https://books.google.co.uk/books/about/Psychometric_Theory.html?id=rOfuAAAAMAAJ\&source=kp_book_descripti on\&redir_esc =y (Accessed: 5 November 2018).

Oesterreich, T. D. and Teuteberg, F. (2016) 'Understanding the implications of digitisation and automation in the context of Industry 4.0: A triangulation approach and elements of a research agenda for the construction industry', Computers in Industry. Elsevier, 83, pp. 121-139. doi: 10.1016/J.COMPIND.2016.09.006.

Palmarini, R. et al. (2018) 'A systematic review of augmented reality applications in maintenance', Robotics and Computer-Integrated Manufacturing. Pergamon, 49, pp. 215-228. doi: 10.1016/J.RCIM.2017.06.002.

Panneta, K. (2018) Gartner Top 10 Strategic Technology Trends for 2019.

Pratama, L. A. and Dossick, C. S. (2019) 'Workflow in Virtual Reality Tool Development for AEC Industry', in Advances in Informatics and Computing in Civil and Construction Engineering. Cham: Springer International Publishing, pp. 297-306. doi: 10.1007/978-3-030-00220-6_36.

Prescott, C. (2018) 'Dataset: Business enterprise research and development (2006-2017)'. Office for National Statistics. Available at:

https://www.ons.gov.uk/economy/governmentpublicsectorandtaxes/researchanddevelopmentexpenditure/datasets/ukb usinessenterpriseresearchanddevelopment.

Ramírez, H. et al. (2015) 'Application of Augmented Reality in Statistical Process Control, to Increment the Productivity in Manufacture', Procedia Computer Science. Elsevier, 75, pp. 213-220. doi: 10.1016/J.PROCS.2015.12.240.

Rekapalli, P. V. and Martinez, J. C. (2011) 'Discrete-Event Simulation-Based Virtual Reality Environments for Construction Operations: Technology Introduction', Journal of Construction Engineering and Management, 137(3), pp. 214-224. doi: 10.1061/(ASCE)CO.1943-7862.0000270.

Research and Markets (2018) Augmented Reality and Virtual Reality Market by Offering (Hardware \&amp; Software), Device Type (HMD, HUD, Handheld Device, Gesture Tracking), Application (Enterprise, Consumer, Commercial, Healthcare, Automotive), and Geography-Global Forecast to 2023. Available at: https://www.researchandmarkets.com/research/qq837j/global_augmented?w=4 (Accessed: 7 February 2019).

Roach, D. M. and Demirkiran, I. (2017) 'Computer aided drafting virtual reality interface', in 2017 IEEE/AIAA 36th Digital Avionics Systems Conference (DASC). IEEE, pp. 1-13. doi: 10.1109/DASC.2017.8102142.

Schall, G. et al. (2009) 'Handheld Augmented Reality for underground infrastructure visualization', Personal and Ubiquitous Computing. Springer-Verlag, 13(4), pp. 281-291. doi: 10.1007/s00779-008-0204-5. 
Schall, G., Mendez, E. and Schmalstieg, D. (2008) 'Virtual redlining for civil engineering in real environments', in 2008 7th IEEE/ACM International Symposium on Mixed and Augmented Reality. IEEE, pp. 95-98. doi: 10.1109/ISMAR.2008.4637332.

Schubert, G. et al. (2015) 'Tangible Mixed Reality On-Site: Interactive Augmented Visualisations from Architectural Working Models in Urban Design', in. Springer, Berlin, Heidelberg, pp. 55-74. doi: 10.1007/978-3-662-47386-3_4.

Seo, D. W. and Lee, J. Y. (2013) 'Physical query interface for tangible augmented tagging and interaction', Expert Systems with Applications. Pergamon, 40(6), pp. 2032-2042. doi: 10.1016/J.ESWA.2012.10.020.

Shi, Y. et al. (2019) 'Impact assessment of reinforced learning methods on construction workers' fall risk behavior using virtual reality', Automation in Construction. Elsevier, 104, pp. 197-214. doi: 10.1016/J.AUTCON.2019.04.015.

Skulmowski, A. et al. (2016) 'Embodied learning using a tangible user interface: The effects of haptic perception and selective pointing on a spatial learning task', Computers \& Education. Pergamon, 92-93, pp. 64-75. doi: 10.1016/J.COMPEDU.2015.10.011.

Turkan, Y. et al. (2017) 'Mobile augmented reality for teaching structural analysis', Advanced Engineering Informatics. Elsevier, 34, pp. 90-100. doi: 10.1016/J.AEI.2017.09.005.

US-Labor-Bureau (2019) 'Job openings levels and rates by industry and region, seasonally adjusted'. Washington DC: U.S. Bureau of Labor Statistics. Available at: https://www.bls.gov/news.release/jolts.t01.htm.

Vähä, P. et al. (2013) 'Extending automation of building construction - Survey on potential sensor technologies and robotic applications', Automation in Construction. Elsevier, 36, pp. 168-178. doi: 10.1016/J.AUTCON.2013.08.002.

Webster, A. et al. (1996) 'Augmented reality in architectural construction, inspection and renovation', in Proc. ASCE Third Congress on Computing in Civil Engineering. Available at: https://www.researchgate.net/profile/Blair_Macintyre/publication/2826659_Augmented_Reality_in_Architectural_Co nstruction_Inspection_and_Renovation/links/0f31753c52918b1309000000.pdf (Accessed: 30 January 2019).

Whyte, J. et al. (2000) 'From CAD to virtual reality: modelling approaches, data exchange and interactive 3D building design tools', Automation in Construction. Elsevier, 10(1), pp. 43-55. doi: 10.1016/S0926-5805(99)00012-6.

Whyte, J. and Nikolic, D. (2018) Virtual Reality and the Built Environment. Second Edi. Routledge. doi: $10.4324 / 9780080520667$

Wolfartsberger, J. (2019) 'Analyzing the potential of Virtual Reality for engineering design review', Automation in Construction. Elsevier, 104, pp. 27-37. doi: 10.1016/J.AUTCON.2019.03.018.

Woyke, E. (2016) ‘Augmented Reality Could Speed Up Construction Projects', MIT Technology Review.

Yiannakopoulou, E. et al. (2015) 'Virtual reality simulators and training in laparoscopic surgery', International Journal of Surgery. Elsevier, 13, pp. 60-64. doi: 10.1016/J.IJSU.2014.11.014.

Young, T.-C. and Smith, S. (2016) ‘An Interactive Augmented Reality Furniture Customization System', in. Springer, Cham, pp. 662-668. doi: 10.1007/978-3-319-39907-2_63.

Yung, R. and Khoo-Lattimore, C. (2017) 'New realities: a systematic literature review on virtual reality and augmented reality in tourism research', Current Issues in Tourism. Routledge, pp. 1-26. doi: $10.1080 / 13683500.2017 .1417359$

Zhao, D. and Lucas, J. (2015) 'Virtual reality simulation for construction safety promotion', International Journal of Injury Control and Safety Promotion. Taylor \& Francis, 22(1), pp. 57-67. doi: 10.1080/17457300.2013.861853.

Zhou, Y., Luo, H. and Yang, Y. (2017) 'Implementation of augmented reality for segment displacement inspection during tunneling construction', Automation in Construction. Elsevier, 82, pp. 112-121. doi:

10.1016/J.AUTCON.2017.02.007. 